\title{
Estratigrafía del Noreste de México y su relación con los yacimientos estratoligados de fluorita, barita, celestina y Zn-Pb
}

\author{
Francisco González Sánchez ${ }^{1,2, *}$, Rafael Puente Solís ${ }^{1,2,}$ Eduardo González Partida², \\ Antoni Camprubín 2,3
}

\author{
${ }^{1}$ Posgrado en Ciencias de la Tierra, Universidad Nacional Autónoma de México. \\ Ciudad Universitaria, Delegación Coyoacán, 04510 México, D.F., México. \\ ${ }^{2}$ Centro de Geociencias, Universidad Nacional Autónoma de México. Campus Juriquilla, \\ Carretera Querétaro-San Luís Potosí km 15.5, Apartado Postal 1-742, 76230 Santiago de Querétaro, Qro., México. \\ ${ }^{3}$ Dirección actual: Instituto de Geofísica, Universidad Nacional Autónoma de México. \\ Ciudad Universitaria, Delegación Coyoacán, 04510 México, D.F., México. \\ *fgonzalez@geociencias.unam.mx
}

\begin{abstract}
Resumen
En el Noreste de México, en los estados de Coahuila y Nuevo León, se encuentran más de 200 depósitos estratoligados conocidos de celestina, barita, fluorita, y Pb-Zn, cuyas características geológicas permiten considerarlos como depósitos de tipo Mississippi Valley (MVT) y similares. Su emplazamiento se produjo en diferentes formaciones de la Cuenca de Sabinas (de edad mesozoica) a partir del inicio de la Orogenia Larámide. Dicha cuenca estuvo limitada por pilares tectónicos (e. g. Bloque de Coahuila) que actuaron como elementos paleogeográficos importantes durante el Mesozoico y controlaron la sedimentación de diversas formaciones detríticas proximales. Para poder explicar la formación de depósitos MVT en el área se realiza la descripción de la estratigrafía de la Cuenca de Sabinas, desde la primera transgresión marina durante el Calloviano, y recogiendo las subsiguientes regresiones y transgresiones durante el Jurásico Superior hasta el Cretácico Superior (Campaniano-Maastrichtiano), en que la Orogenia Larámide cambió los regímenes de sedimentación para esta área. El análisis de la distribución espacial y temporal de los depósitos MVT del Noreste de México pone de manifiesto la estrecha relación entre la presencia de dichos depósitos con secuencias estratigráficas específicas, especialmente las que incluyen evaporitas y carbonatos de ambientes lagunares someros. De la misma manera, se determina que existe una predominancia de depósitos de celestina en áreas que descansan sobre el basamento granítico de edad Permo-Triásica, y la mayoría de yacimientos de fluorita se restringen a zonas cercanas a un basamento predominantemente de metasedimentos también de edad Permo-Triásica. La distribución y agrupamiento de las diferentes mineralizaciones permitieron delimitar y proponer una nueva provincia metalogenética, la "Provincia MVT del Noreste de México" o PMNM y esta, a su vez, subdividirla en cuatro subprovincias, (1) Subprovincia Sur de Celestina, (2) Subprovincia Centro de Plomo-Zinc, (3) Subprovincia Centro de Barita, y (4) Subprovincia Norte de Fluorita. Se sugiere que el $\mathrm{Sr}$ y el Ba de los cuerpos de celestina y barita fueron lixiviados de las formaciones clásticas cuyas áreas fuente son los granitoides permo-triásicos en altos de basamento que limitaron la Cuenca de Sabinas. Así, buena parte del Sr sería de origen radiogénico, lixiviado de formaciones clásticas proximales a altos de basamento, y la formación de los depósitos estratoligados se debería a la interacción de salmueras de cuenca (cuyo flujo habría sido detonado por la Orogenia Larámide) con horizontes estratigráficos favorables a su emplazamiento. Dichas hipótesis constituyen la línea de partida de estudios geoquímicos pertinentes para establecer el modelo deposicional de los depósitos estratoligados de la PMNM.
\end{abstract}

Palabras clave: Depósitos MVT, Cuenca de Sabinas, Noreste de México, estratigrafía del Mesozoico, Orogenia Larámide, salmueras de cuenca. 


\begin{abstract}
In Northeastern Mexico, basically the states of Coahuila and Nuevo León, over 200 known stratabound celestine, barite, fluorite, and $P b$-Zn deposits are found, with geological characteristics that allow to adscribe them to the Mississippi Valley type (MVT) and similar deposits. These deposits occur in different sedimentary formations in the Mesozoic Sabinas Basin since the beginning of the Laramide Orogeny. This basin was bounded by several horsts (i. e. the Coahuila Block) that were important paleogeographic highs during the Mesozoic and ruled the sedimentation of several detritic formations in their vicinities. In order to characterize the formation of MVT in this area we first describe the stratigraphy of the Sabinas Basin, from the first marine transgression during the Callovian, and the subsequent regressions and transgressions during the Upper Jurassic until the Upper Cretaceous (Campanian-Maastrichtian), when the Laramide Orogeny changed the depositional regimes in the region. Our analysis on the space and time distribution of the MVT deposits in Northeastern illustrates the close relationship between the occurrence of such deposits and specific stratigraphic sequences, especially those that contain evaporites and carbonate rocks formed in shallow lagoonal environments. Likewise, we determined the dominance of celestine deposits in rocks overlying the Permian-Triassic granitic basement, and that most of the fluorite deposits are found in neighboring areas to the metasedimentary basement, also aged Permian-Triassic. The distribution and grouping of the different mineralizations allow to set limits to a newly proposed metallogenic province, named "MVT Province of Northeastern Mexico" or MPNM, thus subdivided into four subprovinces, (1) Southern Celestine Subprovince, (2) Central Lead-Zinc Subprovince, (3) Central Barite Subprovince, and (4) Northern Fluorite Subprovince. We also suggest that the Sr and Ba in celestine and barite deposits were leached from clastic formations whose source areas are Permian-Triassic granitic rocks in the basement highs on the borders of the Sabinas Basin. Thus, most Sr would be radiogenic and would have been leached from clastic formations in the vicinities of basement highs, and the formation of stratabound deposits would be due to the interaction of basinal brines (whose flow would have been activated by the Laramide orogeny) with stratigraphic horizons favorable to their circulation. Such hypotheses are the startline to forthcoming geochemical studies to set a depositional model to stratabound deposits in the MPNM.
\end{abstract}

Key words: MVT deposits, Sabinas Basin, Northeastern Mexico, Mesozoic stratigraphy, Laramide Orogeny, basinal brines.

\section{Introducción}

La evolución tectono-sedimentaria del noreste de México y, en específico, el área ocupada por el denominado Golfo de Sabinas, ha estado ligada de una manera muy estrecha a la apertura y desarrollo del Golfo de México (GdM). Los cambios eustáticos que han regido durante la formación del GdM se han manifestado en el noreste de México como etapas periódicas de transgresiones y regresiones. De esta manera, se propició la depositación de secuencias sedimentarias compuestas principalmente de depósitos siliciclásticos de ambientes costeros, depósitos evaporíticos de sabkha, y depósitos de carbonatos tanto de ambientes de plataforma somera y profunda como en complejos arrecifales. Tales secuencias fueron depositadas ininterrumpidamente desde el Jurásico Medio hasta el Cretácico Superior, tiempo en el que la Orogenia Larámide detonó el cambio en el régimen de depósito para esta zona.

Los ambientes de depósito y las condiciones paleogeográficas de esta área, en conjunto con la participación de un evento orogénico, generaron los elementos necesarios para la formación de yacimientos minerales estratoligados semejantes a los depósitos conocidos como Mississippi Valley-Type (MVT), nombre asignado para los yacimientos de plomo-zinc estudiados por primera vez en el Valle del Mississippi. Éstos están definidos por Leach y Sangster (1993) como "Una familia de minerales epigenéticos precipitados a partir de densas salmueras de cuenca, en rangos de temperatura entre $75^{\circ} \mathrm{Ca} 200^{\circ} \mathrm{C}$, depositados en secuencias carbonatadas de plataforma y que carecen de afinidades genéticas relacionadas a actividad ígnea". Los depósitos estratoligados del Noreste de México presentan varias de las características definidas por diversos autores como características diagnósticas de depósitos tipo MVT (Ohle, 1959; Kisvaransayi et al., 1983; Sangster, 1983; Sverjensky, 1986, entre otros), dentro de las cuales las más importantes son: (1) la mayoría de estos depósitos se encuentran alojados en rocas carbonatadas, (2) éstos tienden a localizarse en o cerca de las orillas de cuencas sedimentarias o sobre pilares tectónicos o arcos entre tales cuencas, (3) depósitos individuales semejantes unos a otros pueden estar distribuidos en zonas de varios $\mathrm{km}^{2}$ de extensión, (4) las rocas carbonatadas que los contienen no se encuentran metamorfizadas, (5) se formaron a poca profundidad (generalmente menos de $1500 \mathrm{~m}$ ), (6) existen evidencias de disolución de la roca carbonatada hospedante, con la formación de brechas y texturas de colapso, (7) consisten en reemplazamientos bandeados, geodas o filones, pero siempre estrictamente delimitados a un solo horizonte estratigráfico, (8) presentan una mineralogía 
relativamente simple, y a menudo son monominerálicos, (9) los depósitos están relacionados en tiempo a un evento orogénico, (10) son epigenéticos, estratoligados y los cuerpos presentan con frecuencia relictos de la misma roca hospedante, orientados en forma paralela a la orientación de los estratos, y (11) las brechas y vetillas están cementadas con la misma mineralización que conforma el cuerpo mineralizado. Algunos de los yacimientos del Noreste de México ya han sido tipificados como pertenecientes al tipo MVT (e. g. Buenavista al norte de Coahuila; GonzálezPartida et al., 2003), están formados por celestina, barita, fluorita y sulfuros de plomo y zinc, y su emplazamiento está controlado por formaciones evaporíticas y carbonatadas. De ahí la importancia de determinar su distribución espacial y temporal, así como definir el posible potencial con base en la distribución geográfica de las formaciones sedimentarias que los contienen.

Aunque México ha sido un importante productor de plomo-zinc, muy poco de esta producción fue obtenida de este tipo de yacimientos, los cuales fueron explotados principalmente entre las décadas de 1950 y 1970 . Ejemplos de yacimientos de $\mathrm{Zn}-\mathrm{Pb}$ de esta tipología en el noreste de México son los de Sierra Mojada y Reforma en Coahuila, y El Diente en Nuevo León. En cuanto a los minerales no metálicos, México ocupa una posición preeminente entre los principales productores en el mundo, pues en la actualidad constituye el quinto productor de barita, el segundo de fluorita, y el primero de celestina. La principal zona productora de barita, celestina y fluorita de México es su porción Noreste, debido a la explotación de los yacimientos estratoligados que, en su conjunto, son objeto del presente estudio. El área de estudio cubre casi la totalidad del estado de Coahuila y pequeñas porciones del estado de Nuevo León y del norte del estado de Zacatecas. La superficie que abarca es de aproximadamente 150,000 $\mathrm{km}^{2}$ (Figura 1).

El potencial económico de los depósitos de tipo MVT en la porción Noreste de México sigue siendo muy grande. Sin embargo, se trata de un conjunto de centenares de depósitos que históricamente no han merecido la atención académica debida, tanto a nivel de tipología como a nivel regional. Mediante el presente estudio se pretende establecer las principales características regionales de este tipo de depósitos, a partir de (1) la descripción unitaria de su estratigrafía a partir de diversas fuentes, (2) la reconstrucción de las cuencas Mesozoicas de la región a cuya evolución se asocia la formación de depósitos MVT, (3) la identificación pertinente de todos los depósitos de la región adscribibles a la tipología MVT o similares, y (4) la caracterización a nivel regional de los diferentes subtipos mineralógicos de los depósitos de esta tipología. De esta forma, se pretende sentar el contexto geológico adecuado para estudiar la metalogenia de depósitos individuales de este tipo en el Noreste de México y, a la vez, contribuir al entendimiento de la metalogenia regional asociada a la Orogenia Larámide.

\section{Estructura de las cuencas mesozoicas del Noreste de México}

La configuración estructural y estratigráfica actualmente reconocible de esta región (Figura 2) inicia con el evento orogénico Ouachita-Marathon durante el Permo-Triásico (Goldhammer, 1999) y, a partir del mismo, la evolución paleogeográfica desde el Mesozoico hasta el Cenozoico del Noreste de México se relaciona estrechamente con la apertura y evolución del Golfo de México (Salvador y Green, 1980; Anderson y Schmidt, 1983; Winker y Buffler, 1988; Wilson, 1990). El origen del Golfo de México se remonta al rompimiento del supercontinente Pangea durante el Triásico Superior - Jurásico Medio, con la separación de las placas Norteamericana, Sudamericana y Africana (Padilla y Sánchez, 1986b). Ello condicionó la evolución estratigráfica de la región durante el Cretácico, hasta la Orogenia Larámide, entre el Cretácico Superior al Terciario Inferior (Goldhammer, 1999).

El rompimiento y separación de Pangea propició la formación de pilares y fosas tectónicas que contribuyeron a la distribución de altos y bajos estructurales que, a su vez, controlaron en adelante los patrones sedimentarios de la región (Padilla y Sánchez. 1982b), y posteriormente determinaron los estilos estructurales larámides (Wilson, 1990).

Los principales elementos tectónicos originados en esta región durante la etapa de rifting comprenden altos de basamento, entre los que destacan el Bloque de Coahuila,

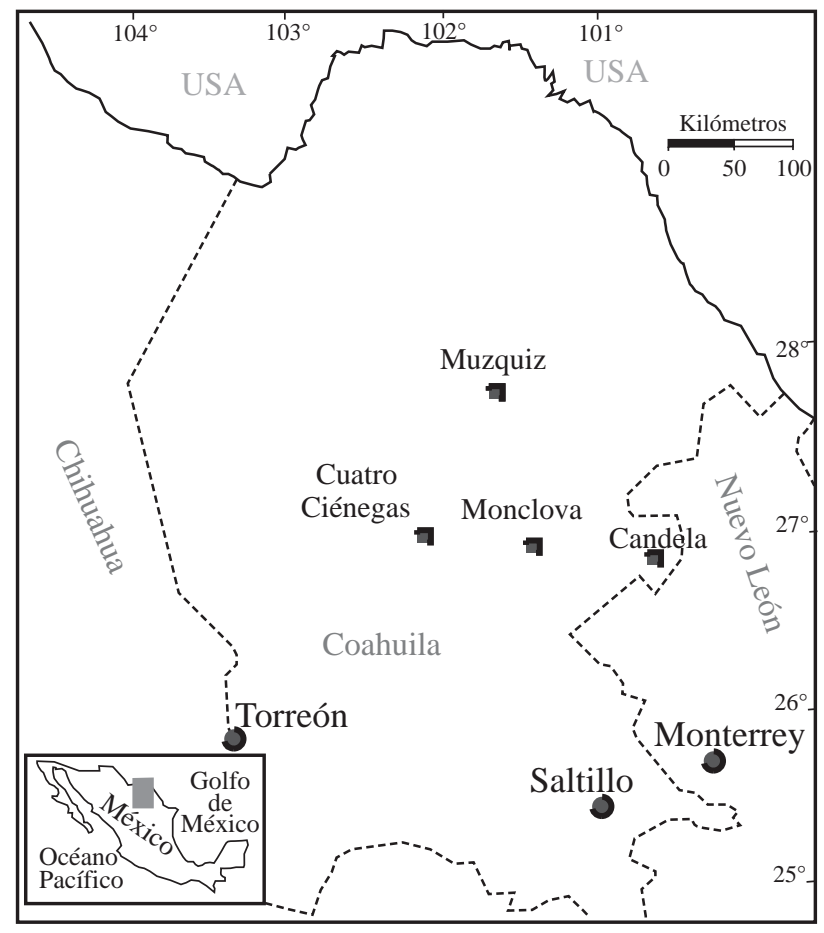

Figura 1. Plano de localización del área de estudio. 


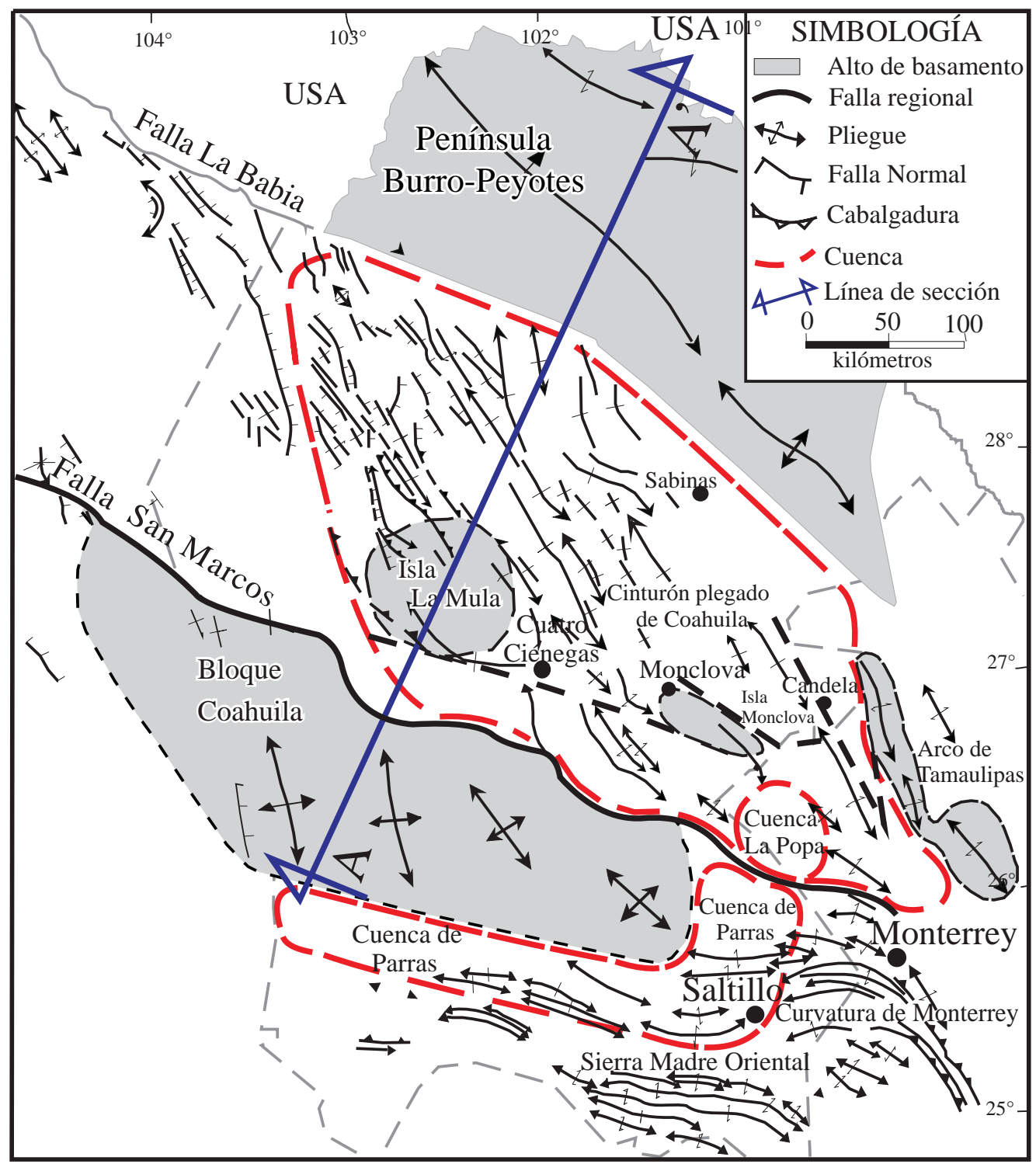

Figura 2. Configuración estructural y rasgos tectónicos del Noreste de México (estado de Coahuila y áreas adyacentes). Modificado de Chávez-Cabello et al. (2005).

la Península de Burro - Peyotes, el Archipiélago de Tamaulipas, y las Islas de La Mula y Monclova, así como bloques bajos de basamento que conforman la Cuenca de Sabinas (Figura 2). La composición litológica de los bloques es diferente de una área a otra debido al acomodo de éstos por movimientos de fallas regionales con desplazamiento lateral.

El Bloque de Coahuila (Figura 2) es un alto de basamento que está intrusionado por rocas graníticas a granodioríticas de edad permo-triásica (Wilson et al., 1984). Hacia el oeste, en el área del Valle Acatita - Las Delicias, el Bloque de Coahuila presenta una sucesión volcanoclástica del Pennsilvánico Medio al Pérmico (McKee et al., 1988; Wilson, 1990). Aunque no aflora su basamento se infiere que probablemente es Proterozoico por las firmas isotópicas de los intrusivos Permo-Triásicos (López et al., 2001). El Bloque de Coahuila está delimitado al norte por una falla lateral izquierda conocida como Falla San Marcos, muy posiblemente activa durante la etapa de rifting del Triásico Tardío al Jurásico Tardío (Charleston, 1981; Chávez-Cabello et al., 2005). Al sur está delimitado por el lineamiento lateral izquierdo Torreón - Monterrey (Anderson y Schmidt, 1983), a lo largo de la Cuenca de Parras. Actualmente, el área ocupada por el Bloque de Coahuila se caracteriza por la presencia de carbonatos de plataforma del Cretácico ligeramente deformados por la Orogenia Larámide (Imlay, 1936; Charleston, 1981; Johnson, 1989; Johnson et al., 1991).

El Archipiélago de Tamaulipas (Figura 2) se localiza al Este de la Cuenca de Sabinas y presenta una distribución NW-SE. El basamento de este arco está constituido por intrusiones permo-triásicas consideradas como los 
remanentes de un arco de islas Paleozoico (Goldhammer, 1999). Su límite oriental lo forma una falla regional lateral derecha, denominada Falla Tamaulipas - Chiapas (Pindell, 1985; Wilson, 1990).

La Península Burro - Peyotes (Figura 2) constituye un alto de basamento al norte de la Cuenca de Sabinas, en aparente continuidad con el Arco de Tamaulipas (Wilson, 1990). Está formado por rocas metasedimentarias deformadas del Paleozoico Superior, y está delimitado en su parte sureste por la Falla La Babia, la cual es una estructura lateral izquierda que estuvo activa durante la etapa de rifting continental del Triásico Superior al Jurásico Medio (Charleston, 1981).

La Cuenca de Sabinas (Figura 2), es una depresión delimitada por los bloques altos de Coahuila al Sur, Burro - Peyotes al Norte, y el Arco de Tamaulipas al Este. Dentro de la cuenca se conocen dos áreas con intrusiones graníticas de edad permo-triásica, estas corresponden a los altos de basamento de La Mula y Monclova (Jones et al., 1984; Wilson, 1990).

La deformación que caracteriza actualmente a la Península Burro - Peyotes, el Arco de Tamaulipas y la Cuenca de Sabinas, incluyendo las Islas de La Mula y Monclova (Figura 2), se conoce como la "Faja Plegada de Coahuila", producida durante la Orogenia Larámide (Goldhammer, 1999). Esta faja plegada consiste en numerosos anticlinales aislados, orientados al NW y separados por amplios valles sinclinales. Anticlinales ramificados, con núcleos formados por diapiros de evaporitas y pliegues con doble vergencia son rasgos frecuentes y característicos de este estilo de deformación (Padilla y Sánchez, 1986b; Goldhammer, 1999).

Otras cuencas aledañas a la Cuenca de Sabinas son las Cuencas de Parras y La Popa (Figura 2) se desarrollaron durante el Cretácico Superior (CampanianoMaastrichtiano). La primera está limitada al norte y noreste por el Bloque de Coahuila y la Falla de San Marcos, y al sur y sureste por el frente de la Sierra Madre Oriental. La Cuenca de La Popa está limitada al norte, este y oeste por la Faja Plegada de Coahuila, y al sur por la Sierra de La Gavia que la separa de la Cuenca de Parras. Ambas cuencas presentan cerca de $5000 \mathrm{~m}$ de sedimentos siliciclásticos terrígenos marinos someros y deltaicos del CampanianoMaastrichtiano pertenecientes al Grupo La Difunta (Padilla y Sánchez, 1986b; Goldhammer, 1999). La deformación que se observa en la actualidad es producto de la Orogenia Larámide. En las porciones sur y este de la Cuenca de Parras, en las zonas próximas al frente de la Sierra Madre, las estructuras son muy alargadas, vergentes hacia el Norte, y presentan pliegues apretados y cabalgaduras menores con ejes paralelos al frente de la sierra. Hacia el norte de estas áreas de la cuenca, la intensidad de la deformación es menor (Goldhammer, 1999). En La Popa, la deformación se caracteriza por amplias elevaciones dómicas generadas por diapiros de sal y sinclinales erosionados (Johnson, 1989).
El evento orogénico del Cretácico Tardío al Paleógeno dió origen a las estructuras de la Sierra Madre Oriental (Figura 2), que se caracteriza por ser el área que presenta el más alto relieve en el Noreste de México. Ésta está limitada al Este por el Arco de Tamaulipas y al Norte por la Cuenca de Parras (Padilla y Sánchez, 1986a). La sección deformada comprende la secuencia sedimentaria del Triásico Superior - Cretácico (Goldhammer, 1999). Las estructuras de esta región se distinguen por su gran variedad de pliegues con orientación E-W, isoclinales, con flancos verticales y pliegues vergentes hacia el Norte, formando incluso nappes, y pueden estar delimitados por cabalgaduras (Padilla y Sánchez, 1986a,b; Johnson, 1989; Eguiluz de Antuñano, 2001).

\section{Estratigrafía del Mesozoico}

A partir de datos indirectos se infiere que en la Cuenca de Sabinas probablemente se depositaron capas rojas de grandes espesores del Jurásico Temprano (Grupo Huizachal) (Goldhammer, 1999; Rueda-Gaxiola et al., 1999; Fastovsky et al., 2005). La acumulación de estas secuencias clásicas de rift probablemente ocurrió en grabens y semigrabens. También se produjo probablemente el emplazamiento de rocas volcánicas calcoalcalinas en las etapas iniciales e intermedias del desarrollo del rift, intercaladas con los conglomerados continentales (Garrison y McMillan, 1999). Posteriormente, ocurrió el depósito de evaporitas que precipitaron durante la primera transgresión marina(Padilla y Sánchez, 1986a,b).

En el transcurso del Calloviano al Oxfordiano Inferior, se depositaron secuencias de transgresión compuestas por conglomerados, evaporitas salinas intercaladas con rocas terrígenas de grano fino, evaporitas anhidríticas y carbonatos. Su espesor en conjunto sobrepasa los $2500 \mathrm{~m}$ (Eguiluz de Antuñano, 2001). En el centro de la cuenca precipitaron principalmente evaporitas (anhidritas, yeso y sal), correspondientes a la Formación Minas Viejas (Eguiluz de Antuñano, 2001) con un espesor original de 1000 m (Goldhammer, 1999). Hacia el Arco de Tamaulipas, la sal tiende a desaparecer y es reemplazada por anhidrita y carbonatos interdigitados hacia la base de la Formación Olvido, y por carbonatos de alta energía hacia su cima, con un espesor de $500 \mathrm{~m}$ en total (Eguiluz de Antuñano, 2001). En las áreas adyacentes a los bloques emergidos de basamento, en cambio, se desarrollan rocas de facies marinas someras compuestas por areniscas cuarzofeldespáticas y conglomerados de la Formación La Gloria (Padilla y Sánchez 1986b; Eguiluz de Antuñano, 2001). Esta formación presenta espesores entre 50 y 100 m, y sobrepasa los $600 \mathrm{~m}$ en las proximidades de los bloques de basamento. Hacia el centro de la cuenca (Figura 3), la Formación La Gloria cambia trancicionalmente hacia arriba a carbonatos de plataforma de la Formación Zuloaga (Oivanki, 1974). 

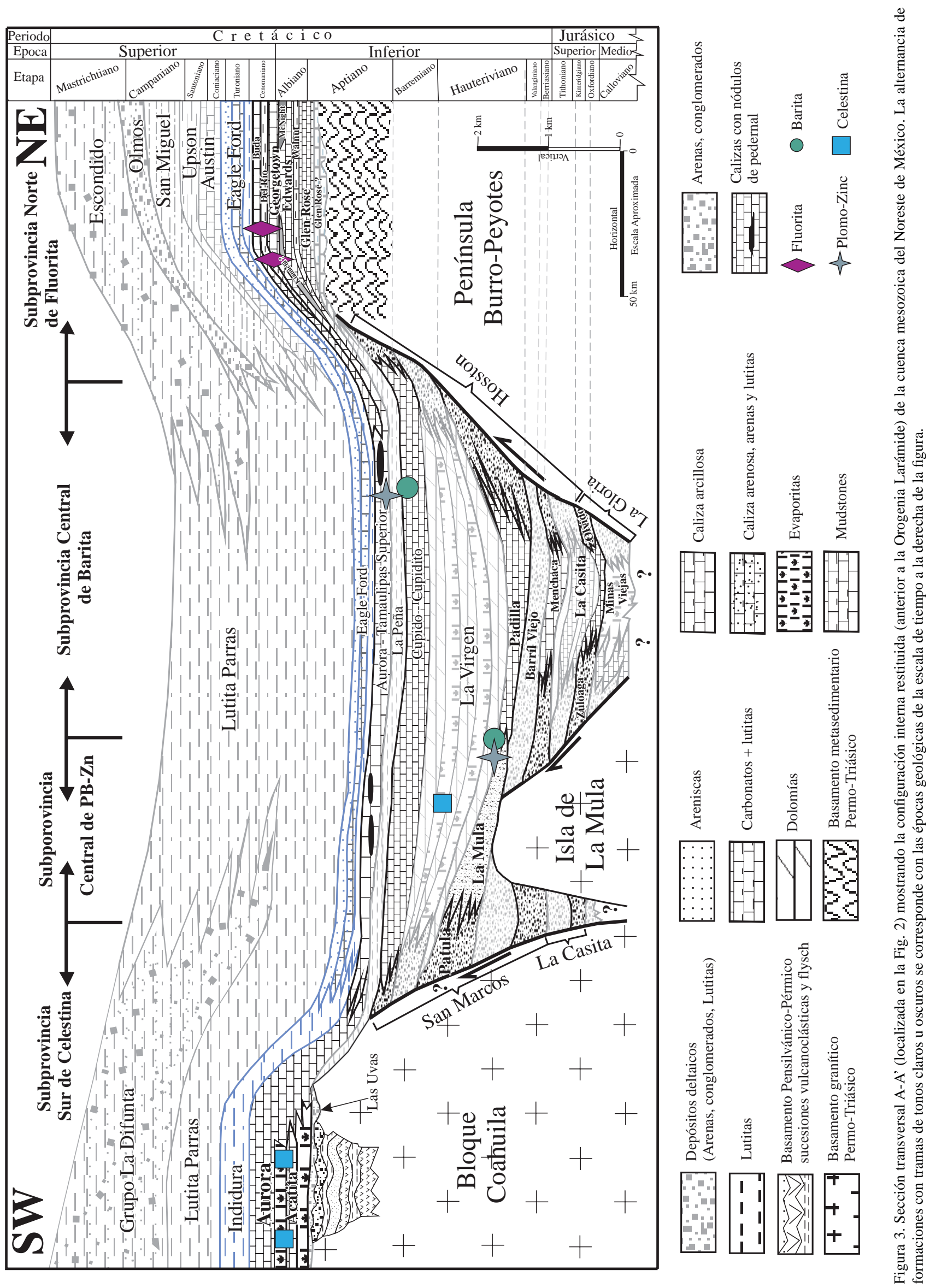
Durante el Oxfordiano Superior, las facies de arenas de playa de la Formación La Gloria (Figura 3) continúan depositándose de forma simultánea, con los carbonatos y evaporitas de la Formación Olvido (Padilla y Sánchez 1986b; Goldhammer, 1999).

Desde el Kimmeridgiano Inferior al Tithoniano, conforme aumenta la subsidencia de la cuenca, las formaciones Gloria y Olvido son cubiertas por lutitas negras de la Formación La Casita (según Eguiluz de Antuñano, 2001) o Grupo La Casita (según Humphrey, 1956). Ésta está integrada por tres miembros, (1) el inferior con lutitas carbonosas, que cambian a facies de arenas costeras hacia los márgenes de la cuenca, (2) el miembro intermedio contiene areniscas, interestratificadas con carbonatos, cuyo tamaño de grano aumenta hacia las orillas de la formación, y (3) el miembro superior formado por lutitas calcáreas negras y limolitas (Eguiluz de Antuñano, 2001). Los espesores de esta formación en su totalidad varían desde 60 hasta 800 m, y su edad abarca desde el Kimmeridgiano hasta la base del Berriasiano (Figura 3).

En el Cretácico Inferior se acumularon sedimentos clásticos marinos someros y marginales gruesos, areniscas y lutitas de la Formación Barril Viejo, con espesores de 250 a $350 \mathrm{~m}$ y una edad correspondiente al Hauteriviano Inferior (Eguiluz de Antuñano, 2001). Hacia el Sureste, esta formación cambia lateralmente a facies de carbonatos de plataforma marina de alta energía, correspondientes a la Formación Menchaca del Berriasiano (Imlay, 1940), con espesores entre 250 y $300 \mathrm{~m}$. Más hacia el Sureste, el cambio lateral de facies prosigue hacia facies de plataforma abierta y facies de cuenca, representadas por lutitas y carbonatos interestratificados de la Formación Taraises (Imlay, 1936; Goldhammer, 1999), con una edad asignada del Berriasiano al Hauteriviano Inferior y espesores que varían entre 135 y $500 \mathrm{~m}$ (Goldhammer, 1999). En las inmediaciones de la Península Burro - Peyotes se depositaron sedimentos terrígenos de la Formación Hosston, en facies fluviales (areniscas y conglomerados) con un rango de edad que abarca desde el Berriasiano hasta el Barremiano Superior. En los alrededores del Bloque de Coahuila y la Isla de La Mula, en cambio, se depositaron conglomerados y areniscas en ambientes terrestres a transicionales y marinos someros de la Formación San Marcos (Eguiluz de Antuñano, 2001), con espesores de $1000 \mathrm{~m}$ (Chávez-Cabello et al., 2005), cuya acumulación es sincrónica a la Formación Hosston (Eguiluz de Antuñano, 2001) (Figura 3).

Durante el intervalo del Hauteriviano Superior al Barremiano, se depositó la Formación Padilla en su facies arrecifal (¿base de la Formación Cupido?), con un espesor promedio de $150 \mathrm{~m}$ (Eguiluz de Antuñano, 2001, y referencias en éste). Hacia el Noreste la Formación Padilla cambia a carbonatos de facies lagunar, con predominio de dolomías. Para este tiempo (Hauteriviano Superior), la Península Burro - Peyotes había sido cubierta en gran parte por la Formación Hosston en su facies de llanura aluvial, con alcance temporal hasta el Barremiano Inferior.
Sincrónicamente, se depositaron sedimentos clásticos de la Formación La Mula hacia el Noroeste de la cuenca, que cubren la Isla de La Mula (Imlay, 1940). Esta formación se adelgaza hacia mar abierto al Este y Sureste de la cuenca (Eguiluz de Antuñano, 2001), y hacia el Bloque de Coahuila grada a areniscas de la Formación Pátula (Figura 3).

Durante el Barremiano Superior se desarrolló el arrecife de la Formación Cupido, cuya formación se extiende hasta mediados del Aptiano. Este arrecife actuó como barrera física, restringiendo la circulación del agua marina hacia la Cuenca de Sabinas, ocasionando la instalación de un ambiente de sabkha y la formación alternada de carbonatos y evaporitas de la Formación La Virgen, con espesores entre 600 y 800 m (Eguiluz de Antuñano, 2001). Esta Formación presenta tres unidades dolomíticas separadas por dos unidades de evaporitas y cambia de facies hacia las Formaciones Hosston y San Marcos hacia el Norte y Sur, respectivamente (Figura 3). Hacia el Este y Sureste del arrecife, se depositaron simultáneamente a la Formación La Virgen carbonatos pelágicos de la Formación Tamaulipas Inferior (Eguiluz de Antuñano, 2001).

El crecimiento del arrecife de la Formación Cupido continuó durante el Aptiano Inferior, mientras que en el lado tras-arrecifal se formaron carbonatos de facies lagunares de alta energía pertenecientes a la Formación Cupidito (Wilson y Pialli, 1977), con un espesor de 250 m acuñándose hacia el Noroeste. Hacia el lado Este del arrecife continuó la deposición de la Formación Tamaulipas Inferior, en tanto que el Bloque de Coahuila (último rasgo en la región que permanecía emergido como isla) fue bordeado por una franja angosta de arenas de playa perteneciente a la Formación San Marcos (Eguiluz de Antuñano, 2001) (Figura 3).

El Aptiano Superior se caracteriza por registrar un incremento en el nivel del mar que cubrió inclusive el Bloque (o Isla) Coahuila. Tal transgresión está representada por las lutitas de la Formación La Peña, que es usada como control bioestratigráfico regional, con espesores variables de hasta $200 \mathrm{~m}$, dependiendo de la topografía anterior a su depósito. Hacia el Sur y Este, esta formación cambia lateralmente a facies de cuenca profunda (lutitas negras y calizas arcillosas con pedernal) de la Formación Otates (Tinker, 1985). En las zonas de menor profundidad de los ya sumergidos bloques de Coahuila y Burro - Peyotes, se depositó la Formación Las Uvas, que consiste en areniscas ricas en carbonatos con espesores de hasta $15 \mathrm{~m}$, descansando discordantemente sobre rocas del basamento (Figura 3; Lehmann et al., 1999; Eguiluz de Antuñano, 2001).

Durante el Albiano-Cenomaniano la subsidencia del área continúa, y ello creó las condiciones para que en la parte oriental de la cuenca se depositaran capas delgadas y gruesas de mudstone a wackestone con pedernal de la Formación Tamaulipas Superior del Albiano, con espesores entre 100 y $200 \mathrm{~m}$. Hacia los bordes de lo que fue la Isla de Coahuila, la Formación Tamaulipas Superior cambia a facies de carbonatos de plataforma de la Formación Aurora (Goldhammer, 1999, y referencias en éste), con 
espesores entre 500 y $700 \mathrm{~m}$ (Vinet, 1975). Sobre el Bloque de Coahuila, la Formación Aurora configuró una laguna en la que se depositó una secuencia que inicia con una caliza masiva de bioclastos, de 60 a $80 \mathrm{~m}$ de espesor, que cambia verticalmente a una alternancia de evaporitas con dolomitas de aproximadamente $500 \mathrm{~m}$ de espesor, asignada a la Formación Acatita de edad Albiano Inferior-Medio (Lehmann et al., 1999, y referencias en éste). La Formación Acatita está cubierta por el miembro superior de la Formación Aurora (Goldhammer, 1999), con espesores de 190 a $260 \mathrm{~m}$ y alcance temporal hasta el Cenomaniano. La Formación Aurora es equivalente a la Formación Georgetown del Albiano Superior a inicios del Cenomaniano, formada por calizas micríticas con nódulos de pedernal y espesores de 80 a 150 m (Eguiluz de Antuñano, 2001). Padilla y Sánchez (1986a,b) menciona que es probable que en el Bloque Burro - Peyotes hayan existido condiciones semejantes a las del Bloque de Coahuila, y que la Formación McKnight sea equivalente a La Formación Acatita (Figura 3).

Hacia la parte Norte-central de la cuenca, simultáneamente a las formaciones arriba mencionadas, se depositaron las Formaciones Del Río y Buda, equivalentes al miembro superior de la Formación Aurora (Figura 3). La Formación Del Río se formó del Cenomaniano Inferior al Medio y contiene lutitas y areniscas de grano fino, con espesores desde $40 \mathrm{~m}$ en sus porciones norte y noreste, y hasta $5 \mathrm{~m}$ hacia su porción sureste. La Formación Buda consiste en lodolitas calcáreas de cuenca, con espesores de 10 a 50 $\mathrm{m}$ y una edad correspondiente al Cenomaniano Superior (Eguiluz de Antuñano, 2001, y referencias en éste).

La Formación Kiamichi (o Formación Sombreretillo según Eguiluz de Antuñano, 2001) consiste en una intercalación de lutitas y calizas. En la Figura 3, esta formación está indicada con una interrogación por el hecho de que existen dudas acerca de su ubicación estratigráfica, ya que Lehmann et al. (1999) y Winker y Buffler (1988) consideran que se ubica sobreyaciendo a la Formación Tamaulipas Superior e infrayaciendo a la Formación Georgetown, pero Humphrey (1956), Ramírez (1966), Aguayo (1978) y Eguiluz de Antuñano (2001) consideran que la Formación Georgetown es sincrónica a las Formaciones Tamaulipas Superior y Aurora.

A fines del Cenomaniano y durante el Turoniano se depositó un paquete de $300 \mathrm{~m}$ de espesor de lutitas negras con calizas arenosas y areniscas calcáreas intercaladas, pertenecientes a la Formación Eagle Ford (Eguiluz de Antuñano, 2001). Hacia las porciones sur y sureste de la cuenca, esta formación cambia lateralmente a facies de plataforma de las Formaciones Indidura y San Felipe, respectivamente (Eguiluz de Antuñano, 2001) (Figura 3).

Durante el intervalo del Coniaciano al Santoniano Medio, el nivel del mar descendió, de tal manera que se incrementó la formación de carbonatos y disminuyó la de lutitas. En este contexto se depositó la Formación Austin (Figura 3), con espesores entre 200 y 300 m. Ésta presenta, además, intercalaciones en capas delgadas de bentonita (Padilla y Sánchez, 1986b; Eguiluz de Antuñano, 2001). La Formación Indidura se depositó en un intervalo de tiempo del Cenomaniano Superior al Santoniano, y es correlacionable con las Formaciones Eagle Ford y Austin en la parte norte de la Cuenca de Sabinas (Goldhammer, 1999).

A partir del Santoniano Medio se empiezan a apreciar los efectos de la Orogenia Larámide, al formarse las cuencas interiores (Área Sabinas - Olmos y las Cuencas Parras y Popa) y al producirse el depósito de formaciones típicas de ambientes de planicies aluviales continentales y complejos deltaicos.

La Formación Upson (Figura 3), depositada durante el Santoniano Superior-Campaniano Medio (Padilla y Sánchez, 1986b), está constituida por lutitas con espesores de 100 a $150 \mathrm{~m}$, formadas en un ambiente de prodelta y correlacionables con la Lutita Parras al sur (Eguiluz de Antuñano, 2001).

La Formación San Miguel (Figura 3), del Campaniano Medio-Maastrichtiano Inferior según Padilla y Sánchez (1986b), o bien del Santoniano Superior al Campaniano Inferior según Eguiluz de Antuñano (2001), está constituida por una alternancia de sedimentos progradacionales arenosos y arcillosos con un espesor de hasta $400 \mathrm{~m}$, formados en un ambiente de frente de delta (Eguiluz de Antuñano, 2001).

La Formación Olmos (Figura 3), del Maastrichtiano Medio según Padilla y Sánchez (1986b), o bien del Campaniano Superior según Eguiluz de Antuñano (2001), consiste de areniscas y contiene importantes yacimientos de carbón. Esta formación es correlacionable con el Grupo La Difunta de la Cuenca de La Popa, cuya edad es Maastrichtiano-Paleoceno (Padilla y Sánchez, 1986b).

Durante el Maastrichtiano Superior, la Formación Escondido acumuló un espesor de $800 \mathrm{~m}$ de areniscas conglomeráticas y carbón en su base (Padilla y Sánchez, 1986b), y se correlaciona con el Grupo La Difunta de la Cuenca de La Popa (Figura 3; Eguiluz de Antuñano, 2001).

\section{Descripción de las mineralizaciones estratoligadas tipo MVT y su distribución espacial y temporal}

Los yacimientos estratoligados identificados como pertenecientes al tipo MVT en el Noreste de México presentan una mineralogía simple, y normalmente son depósitos monominerales de celestina, barita, fluorita, y de $\mathrm{Zn}-\mathrm{Pb} \pm$ barita, con minerales de ganga como yeso tardío, calcita, arcillas, y minerales de uranio en cantidades subordinadas. Los depósitos de celestina, barita y fluorita presentan sus contactos bien definidos con la roca encajonante y no existe una disminución gradual de la mineralización hacia la roca tanto en las mineralizaciones estratoligadas como en las brechas, fracturas y rellenos cársticos. En cuanto a alteraciones hidrotermales, los depósitos identificados 
presentan escasa dolomitización y/o silicificación. Ahora bien, los depósitos de $\mathrm{Zn}-\mathrm{Pb}$, constituídos esencialmente por sulfuros, suelen presentar una acusada alteración supergénica con la consiguiente alteración de los sulfuros a óxidos, carbonatos y sulfatos.

\subsection{Mineralizaciones encajonadas en la Formación La} Virgen

En la base de la Formación La Virgen (Barremiano Inferior) se hospedan los cuerpos minerales estratoligados estratigráficamente más profundos conocidos en la actualidad del Noreste de México. Estos cuerpos están constituidos por barita y sulfuros de $\mathrm{Pb}-\mathrm{Zn}$ (Figura 4) y se localizan casi en el contacto con la Formación La Mula (Figura 5).
Los cuerpos de barita son estratiformes, presentan textura nodular con tamaños de orden milimétrico a centimétrico, y colores entre blanco lechoso y gris claro. Estos depósitos presentan un fuerte fracturamiento (probable evidencia de deformación sinformacional) relleno de sulfuros de $\mathrm{Pb}-\mathrm{Zn}$ con texturas botrioidales y dendríticas (Figura 6), en proceso de oxidación supergénica. En general, la zona mineralizada en la Formación La Virgen se caracteriza por la presencia de fracturas con mineralización de $\mathrm{Pb}-\mathrm{Zn}$, orientadas principalmente al Noroeste.

En la cima de la Formación La Virgen (Barremiano Superior) se alojan cuerpos estratoligados de escasos metros de espesor de celestina con la formación tardía de azufre nativo. La mineralización se presenta en el cambio litológico entre rocas carbonatadas y evaporitas de yeso. Tanto los yacimientos de celestina-azufre como los de

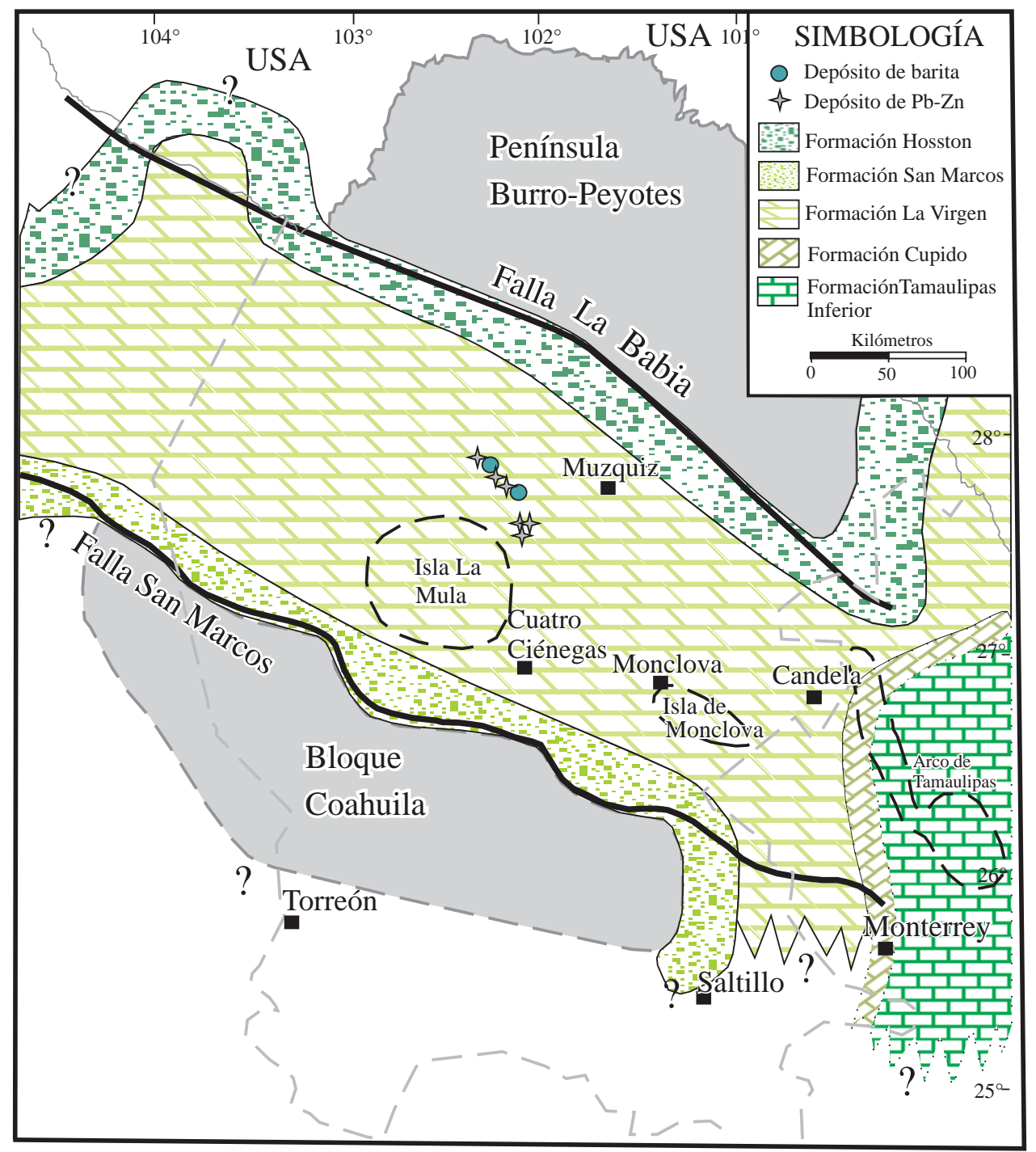

Figura 4. Distribución de las mineralizaciones de plomo-zinc (estrellas) y barita (círculos) en la base de la Formación La Virgen (Barremiano Temprano), con los rasgos paleogeográficos de la época. Modificado de Eguiluz de Antuñano (2001). 

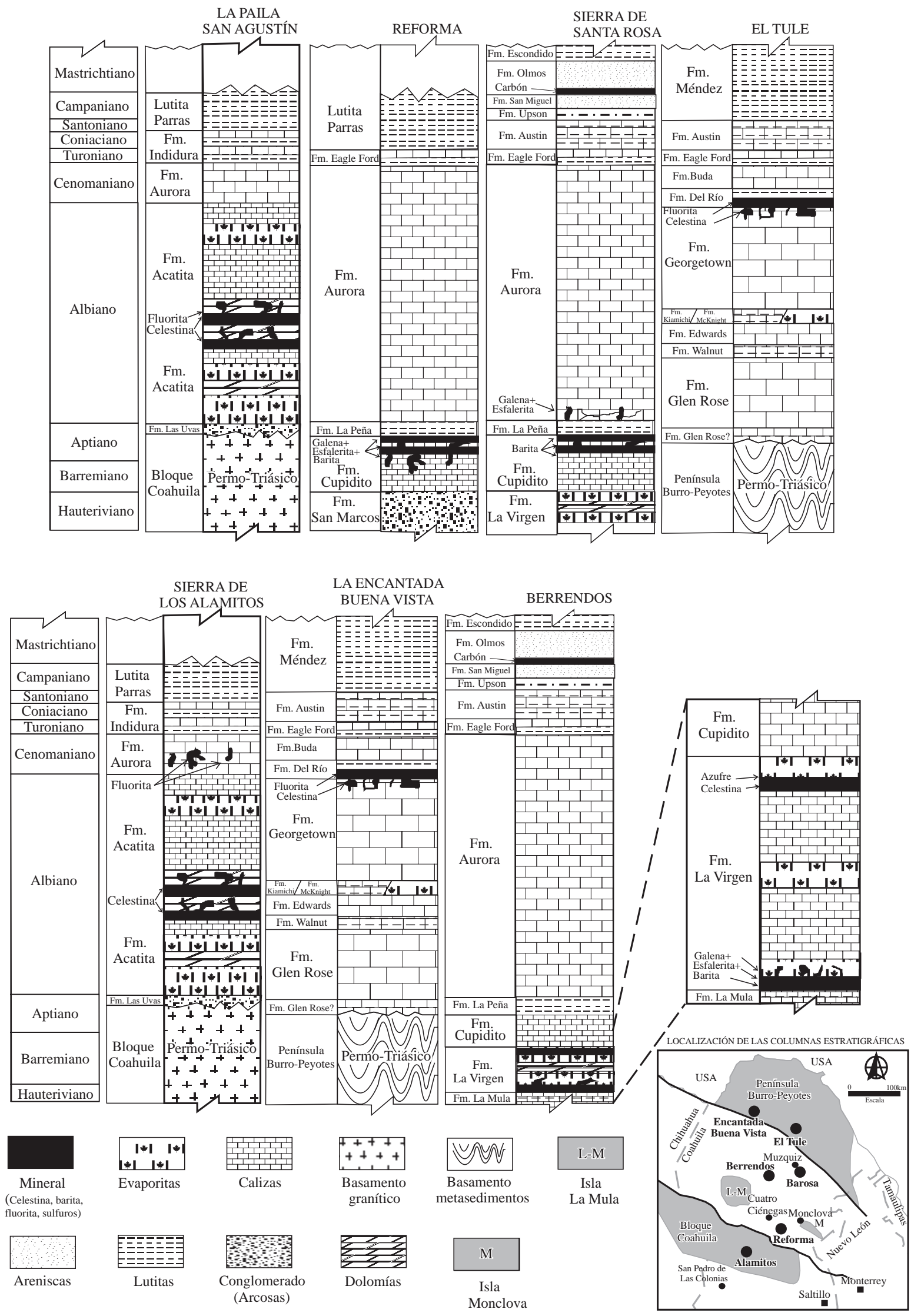

Figura 5. Columnas estratigráficas sintéticas de yacimientos selectos mostrando la distribución vertical de los diferentes tipos de mineralización estratoligada del Noreste de México en la Cuenca de Sabinas y áreas adyacentes. 


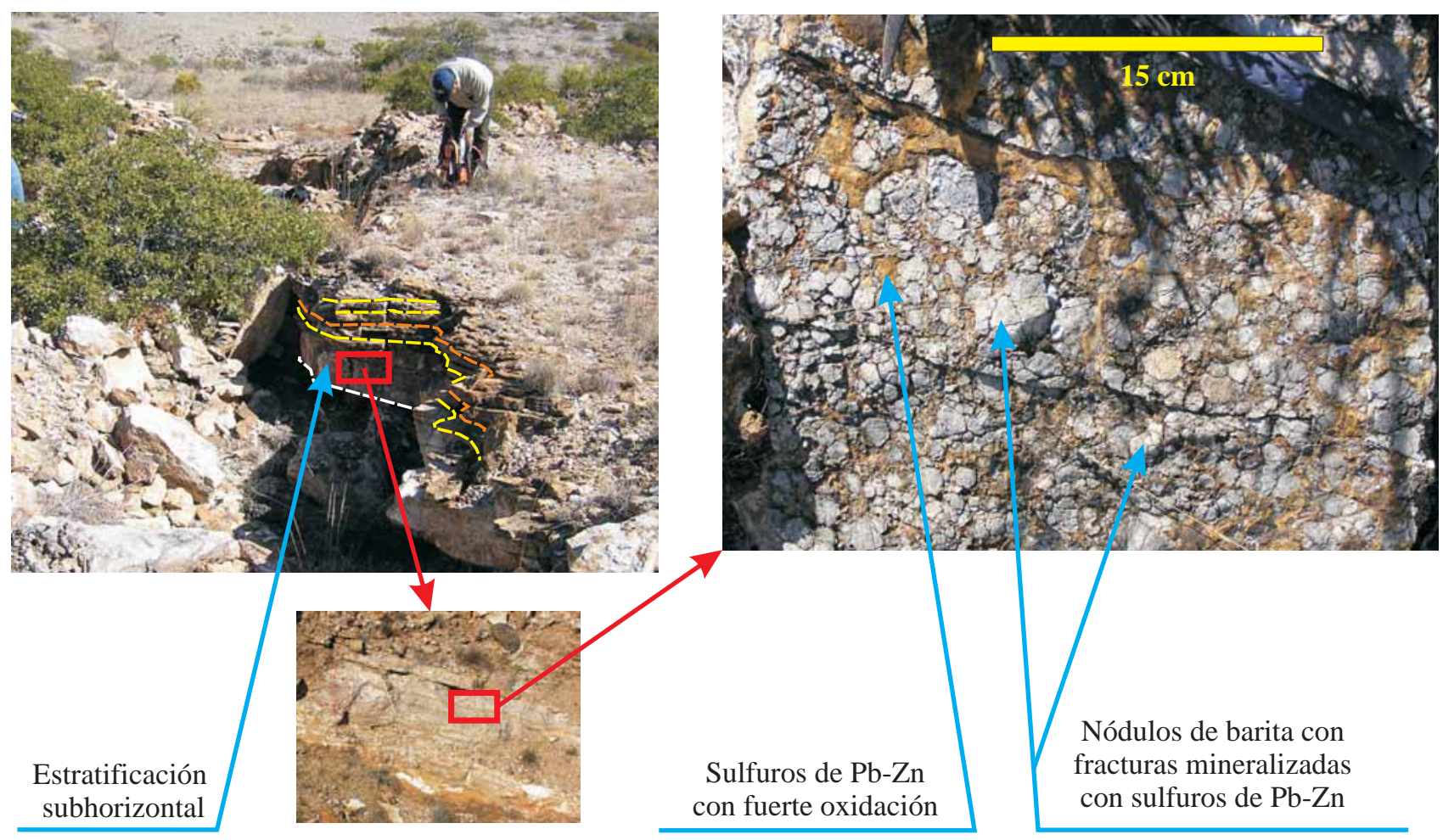

Figura 6. Fotografías mostrando el carácter estratoligado de la mineralización y la textura en nódulos de la barita (derivada posiblemente del reemplazamiento de nódulos de yeso o anhidrita) en los yacimientos de $\mathrm{Pb}-\mathrm{Zn}$-barita de la zona de Berrendos, Coahuila.

barita-Pb-Zn, afloran en el área conocida como Potrero de Berrendos, ubicada en la zona central de la Cuenca de Sabinas (Figuras 5, 7 y 8 ).

\subsection{Mineralizaciones encajonadas en la Formación Cupidito}

Encajonadas en la Formación Cupidito (Aptiano Inferior) se encuentran diversas mineralizaciones de $\mathrm{Pb}-\mathrm{Zn}$ y/o de barita. Los cuerpos de $\mathrm{Pb}-\mathrm{Zn}$ de menor tamaño se presentan en forma de mantos estratoligados, concordantes con la estratificación, mientras que los de mayor tamaño se encuentran en forma de rellenos de paleokarsts. El contacto de las mineralizaciones con las rocas encajonantes es abrupto, aunque cuando los sulfuros se encuentran oxidados o en proceso de oxidación, las rocas encajonantes adoptan tonalidades rojizas y amarillentas aparentando un falso contacto transicional. Encajonados en la Formación Cupidito se encuentran algunos de los yacimientos de $\mathrm{Pb}$ Zn más importantes del área de estudio, como El Diente en Nuevo León, o Sierra Mojada y Reforma en Coahuila. Cabe señalar que la distribución de la Formación Cupidito abarca prácticamente todo el largo y ancho de la Cuenca de Sabinas (Figuras 5 y 9).

La gran mayoría de cuerpos estratoligados de barita explotados hasta la actualidad se restringen principalmente a la parte centro norte de la Cuenca de Sabinas, en la Sierra de Santa Rosa al oriente de Múzquiz, Coahuila (Figura 9). Los niveles estratoligados mineralizados presentan espesores de hasta 3 metros, y la mineralización presenta texturas desde agregados sacaroidales y de reemplazamiento de nódulos, hasta cristales euedrales de orden decimétrico, con la presencia recurrente de texturas rítmicas (Figura 10).

\subsection{Mineralizaciones encajonadas en la Formación Acatita}

Algunas de las áreas con mayor concentración en la región de yacimientos estratoligados de celestina, así como algunos yacimientos de fluorita, son la Sierra de Los Alamitos y la Sierra de La Paila, ubicadas sobre el Bloque de Coahuila, en la parte sur-suroeste del área estudiada (Figura 11). Dichos yacimientos están encajonados en rocas de la Formación Acatita del Albiano (Figura 5).

Las mineralizaciones encajonadas en la Formación Acatita se presentan (1) en forma de mantos reemplazando probablemente rocas evaporíticas, (2) en forma de relleno de cavidades (fracturas y cavernas), o (3) cementando brechas. Dentro de los cuerpos mineralizados es común encontrar relictos de la roca original, incluyendo yeso evaporítico, orientados en concordancia con la estratificación. Los cuerpos estratoligados presentan espesores desde 


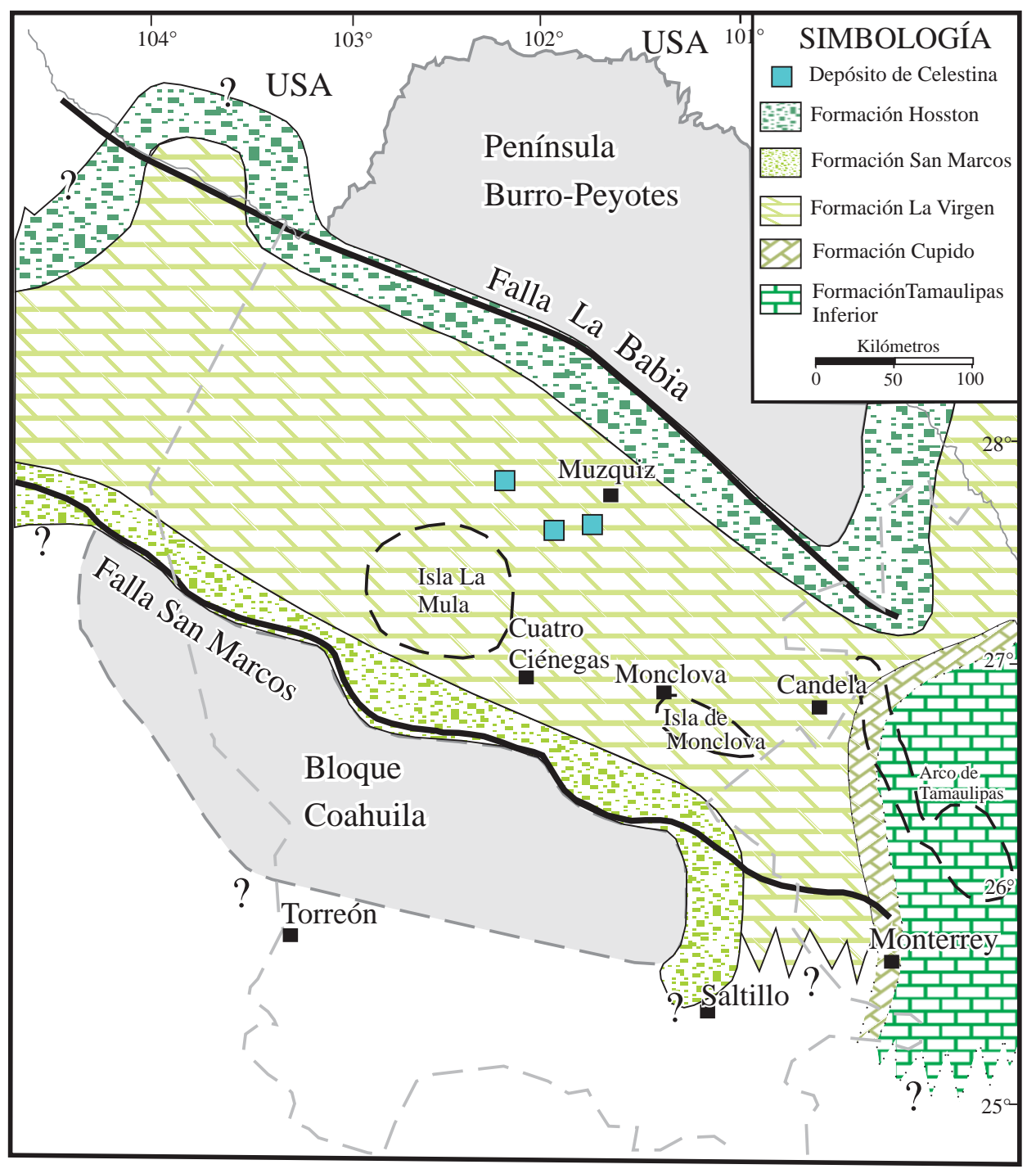

Figura 7. Distribución de las mineralizaciones de celestina (cruces) en la cima de la Formación La Virgen (Barremiano Tardío), con los rasgos paleogeográficos de la época. Modificado de Eguiluz de Antuñano (2001).

escasos centímetros hasta 3 metros o más. Las texturas presentes comprenden, en la zona superior de los cuerpos, grandes cristales euedrales tabulares hasta de orden decimétrico en arreglos en libro y, en la parte inferior de los cuerpos, se presentan casi invariablemente ritmitas. Los contactos entre los cuerpos mineralizantes y las rocas encajonantes son abruptos y, en ocasiones, se observa una incipiente aureola de dolomitización de pocos centímetros de espesor (Figura 12).

\subsection{Mineralizaciones encajonadas en la Formación Buda}

A lo largo del borde de la Península Burro - Peyotes (Figura 13) se encuentra una alta concentración de cuerpos estratoligados de fluorita. En esta zona, las mineralizaciones se encuentran encajonadas en rocas sedimentarias del Grupo
Washita, equivalente al miembro superior de la Formación Aurora, con la particularidad de que las mineralizaciones se hallan preferencialmente emplazadas en el contacto entre las Formaciones Del Río y Buda del Cenomaniano Medio (Figura 5). Sobre esta misma paleopenínsula, se localizan algunos yacimientos de celestina (Figura 13) en forma de mantos coronados por horizontes de fluorita no económicos. Sin embargo, este estilo de mineralización no es exclusivo de esta zona, pues se encuentra también en localidades sobre Bloque de Coahuila como la Sierra de La Paila (distrito minero de San Agustín) y en la Sierra de Los Alamitos (Figura 11).

En la Sierra de Santa Rosa, en Múzquiz, Coahuila, existen pequeñas mineralizaciones subeconómicas de sulfuros de $\mathrm{Pb}-\mathrm{Zn}$ en la base de la Formación Georgetown (equivalente a la Formación Aurora). En esta zona, las mineralizaciones se presentan principalmente como re- 


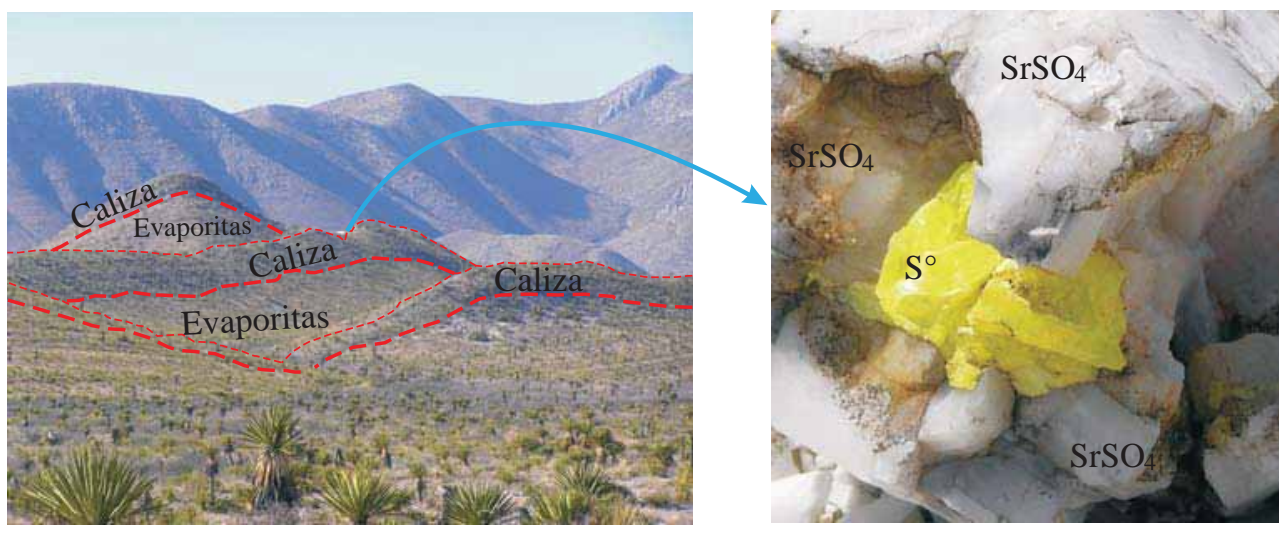

Figura 8. Fotografía panorámica mostrando la Formación La Virgen con su alternancia de caliza-dolomita/evaporitas en el área de Potrero de Berrendos, y la localización de una mineralización de celestina y azufre nativo.

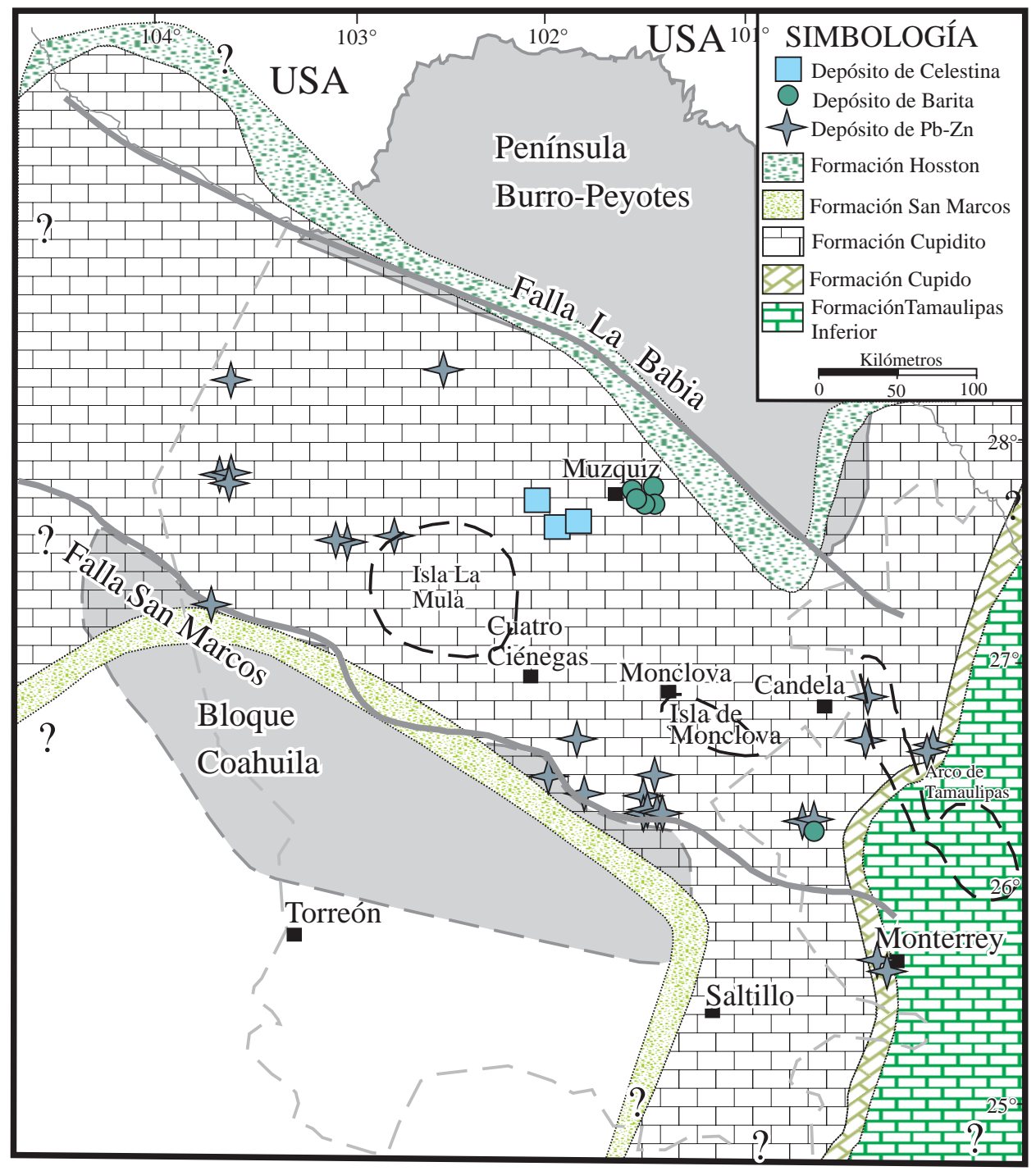

Figura 9. Distribución de la mineralización de Pb-Zn-barita en las Formaciones Cupidito y Cupido (Aptiano), con los rasgos paleogeográficos de la época. Modificado de Eguiluz de Antuñano (2001). 


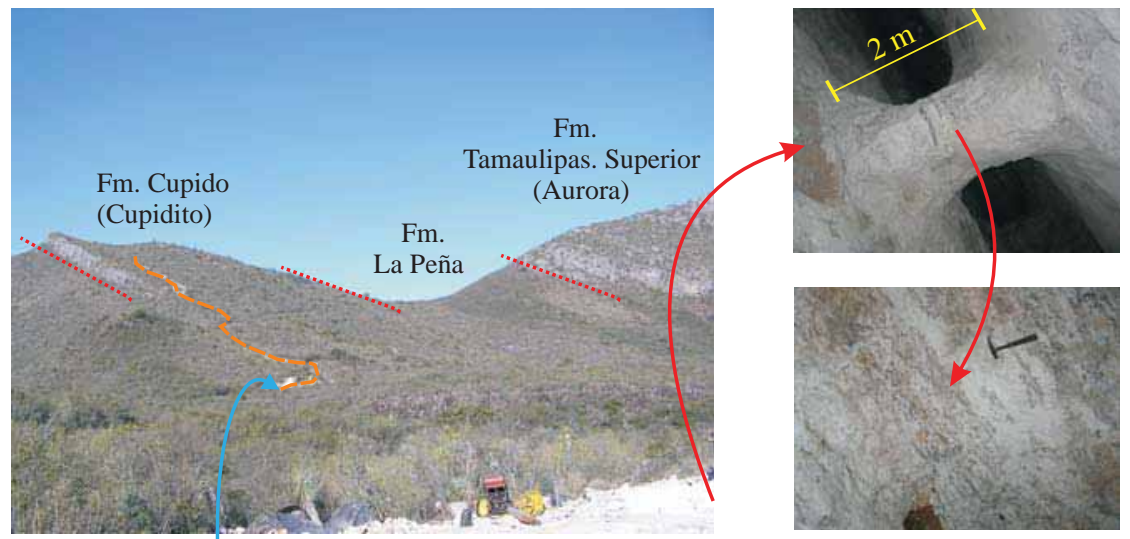

Traza del estrato mineralizado

Figura 10. Mineralización de barita en la Sierra de Santa Rosa, Distrito de Múzquiz, Coahuila. La línea curveada continua en la Formación Cupidito muestra la traza del estrato mineralizado.

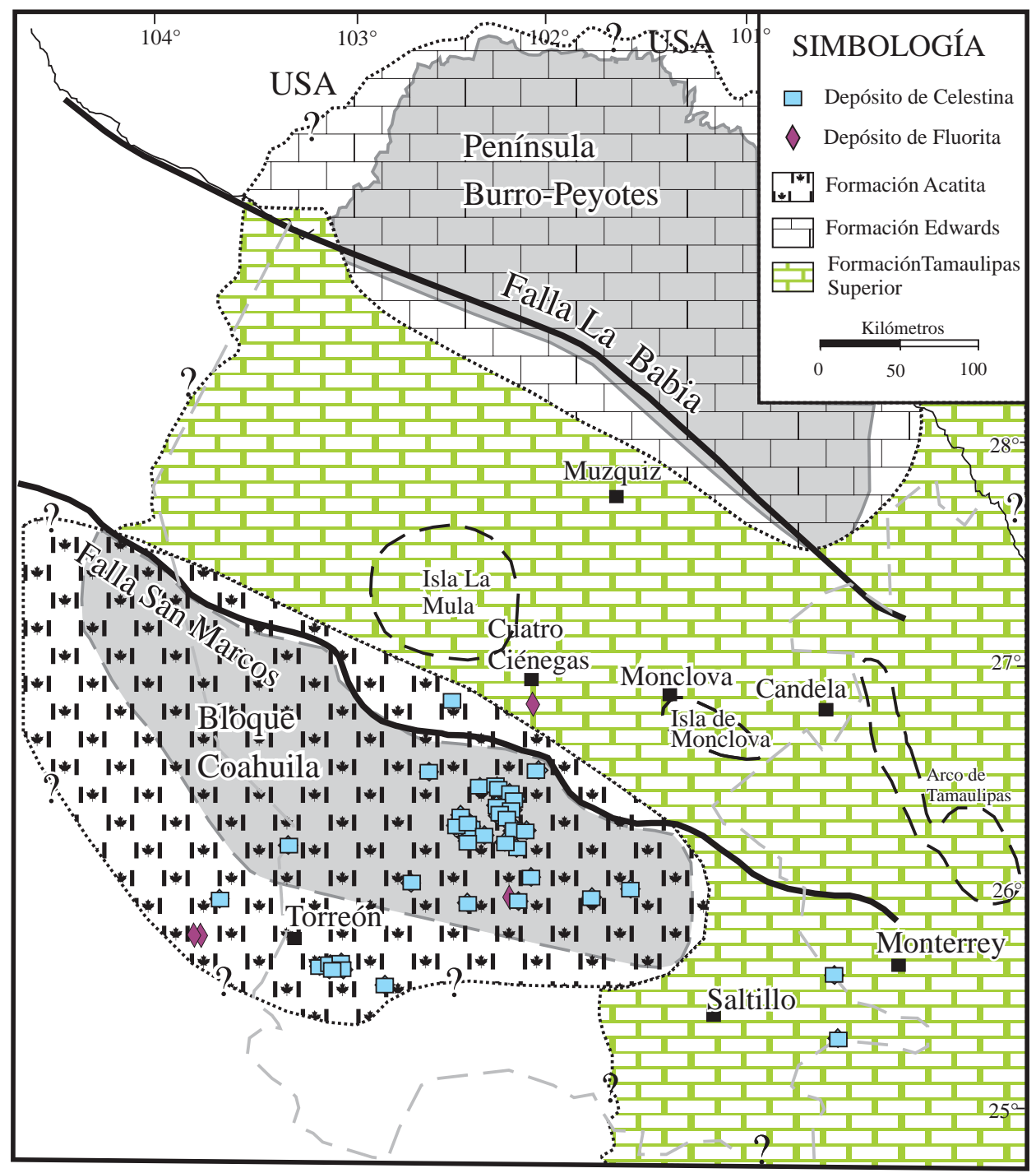

Figura 11. Distribución de las mineralizaciones de celestina (cruces) y fluorita (rombos) en la Formación Acatita (Albiano), con los rasgos paleogeográficos de la época. Modificado de Eguiluz de Antuñano (2001). 
llenos de pequeñas estructuras kársticas y de fracturas de diferente espesor ( 1 a $40 \mathrm{~cm}$ ). Aunque la mineralogía primaria se encuentra generalmente oxidada, se pueden observar relictos de galena.

\section{Discusión}

\subsection{La Provincia metalogenética MVT del Noreste de México.}

Los registros de más de 200 depósitos estratoligados analizados de los archivos del Servicio Geológico Mexicano (Puente-Solís, 2006), muestran que dichos depósitos presentan características que permiten agruparlos como depósitos de tipo Mississippi Valley (MVT). Además, la mayoría de estos depósitos, todos ellos emplazados dentro de la Cuenca Mesozoica del Noreste de México, presentan características texturales y morfológicas comunes. Entre dichas características, las principales son las siguientes.

(1) Los depósitos están alojados en unidades estratigráficas que contienen rocas carbonatadas de plataforma y/o evaporitas, formadas durante las etapas iniciales de transgresiones marinas en fosas tectónicas que describen rasgos paleogeográficos como el Golfo de Sabinas, y en altos de basamento o pilares tectónicos que describen rasgos paleogeográficos como el Bloque de Coahuila, la Isla de La Mula, la Isla de Monclova, el Arco de Tamaulipas y la Península Burro - Peyotes.

(2) Los depósitos están localizados sobre los límites (o en sus proximidades) de los pilares tectónicos constituidos por rocas del basamento, o bien

(3) están localizados en las proximidades a grandes sistemas de fallas que han sido reactivadas en varias épocas, y derivan o son el reflejo de grandes estructuras generadas durante la apertura del Golfo de México.

Dado que estos depósitos fueron formados en ambientes similares, debieron estar también asociados a los mismos procesos geológicos (incluyendo la Orogenia Larámide, que habría detonado el flujo de fluidos asociado), y dado que se encuentran emplazados en una área concreta y en unidades geológicas específicas, es razonable agrupar este conjunto de depósitos en una provincia metalogenética no definida formalmente con anterioridad: la Provincia Metalogenética MVT del Noreste de México (PMNM, Figura 14).

Esta provincia puede, a su vez, ser subdividida en cuatro subprovincias, de sur a norte, (1) la Subprovincia Sur de Celestina, asociada con el Bloque de Coahuila y con el Alineamiento Torreón - Monterrey en La Cuenca de Parras, (2) la Subprovincia Central de Plomo-Zinc, asociada burdamente con la Falla San Marcos en la parte central de la Cuenca de Sabinas, (3) la Subprovincia Central de Barita, localizada en la parte central norte de la
Cuenca de Sabinas, y (4) la Subprovincia Norte de Fluorita, asociada con la Península Burro - Peyotes y la Falla La Babia (Figura 14).

Cada una de las cuatro subprovincias se ha definido por contener la mayoría de los depósitos con características de mineralización o asociaciones similares, por presentar las mineralizaciones estratoligadas encajonadas preferencialmente en secciones estratigráficas concretas (ver apartado 4), y su extensión geográfica no presenta traslapes significativos. Además, la extensión geográfica de cada subprovincia está asociada directamente con elementos tectónicos (pilates o fosas tectónicas) o paleogeográficos (paleoislas o paleopenínsulas) relevantes. La distinta mineralogía de los depósitos estratoligados tipo MVT asociados con los elementos anteriores sugiere que, a pesar que los procesos geológicos generales involucrados en la formación de estos depósitos parecen ser los mismos, hay factores clave adicionales que determinan o influyen sobre la diferente mineralogía de tales grupos de depósitos.

\subsection{Origen de componentes de las mineralizaciones}

Kesler y Jones (1981), en base a estudios isotópicos de Sr en diversos depósitos estratoligados de la región, fueron los primeros en sugerir que el estroncio y el bario de los depósitos de celestina y barita pueden provenir principalmente de las rocas clásticas derivadas del basamento granítico. Sin embargo, a pesar de tratarse de una explicación razonable, la interpretación de dichos autores no se basó más que en las composiciones isotópicas obtenidas, sin sustento en el análisis estratigráfico detallado de la cuenca mesozoica. Las rocas del basamento regional en el Noreste de México son principalmente de edad permo-triásica. Dicho basamento, en la parte sur de la Provincia Metalogenética MVT del Noreste de México (Bloque de Coahuila, Isla de La Mula y Arco de Tamaulipas) contiene predominantemente rocas graníticas, mientras que en la porción norte de la misma (Península Burro - Peyotes) contiene predominantemente rocas metasedimentarias. Ambos tipos de rocas del basamento constituyeron las áreas fuente de los fragmentos detríticos de las rocas clásticas presentes en la cuenca mesozoica de la región. Debido básicamente a su porosidad, tales rocas clásticas (especialmente conglomerados, arcosas y rocas carbonatadas arenosas en Formaciones como la San Marcos y la Hosston) fueron las unidades que con mayor probabilidad pudieron haber actuado como acuíferos que albergaron salmueras de cuenca por periodos de tiempo indeterminado. Estas rocas están compuestas por partículas detríticas provenientes de los elementos tectónicos emergidos para esos tiempos. Para el caso de los detritos derivados de la erosión del Bloque de Coahuila, la Isla de La Mula, la Isla de Monclova y el Arco de Tamaulipas (basamento granítico), las formaciones detríticas que los contienen pudieron haber generado cantidades significativas de estroncio radiogénico, incorporado a las salmueras 


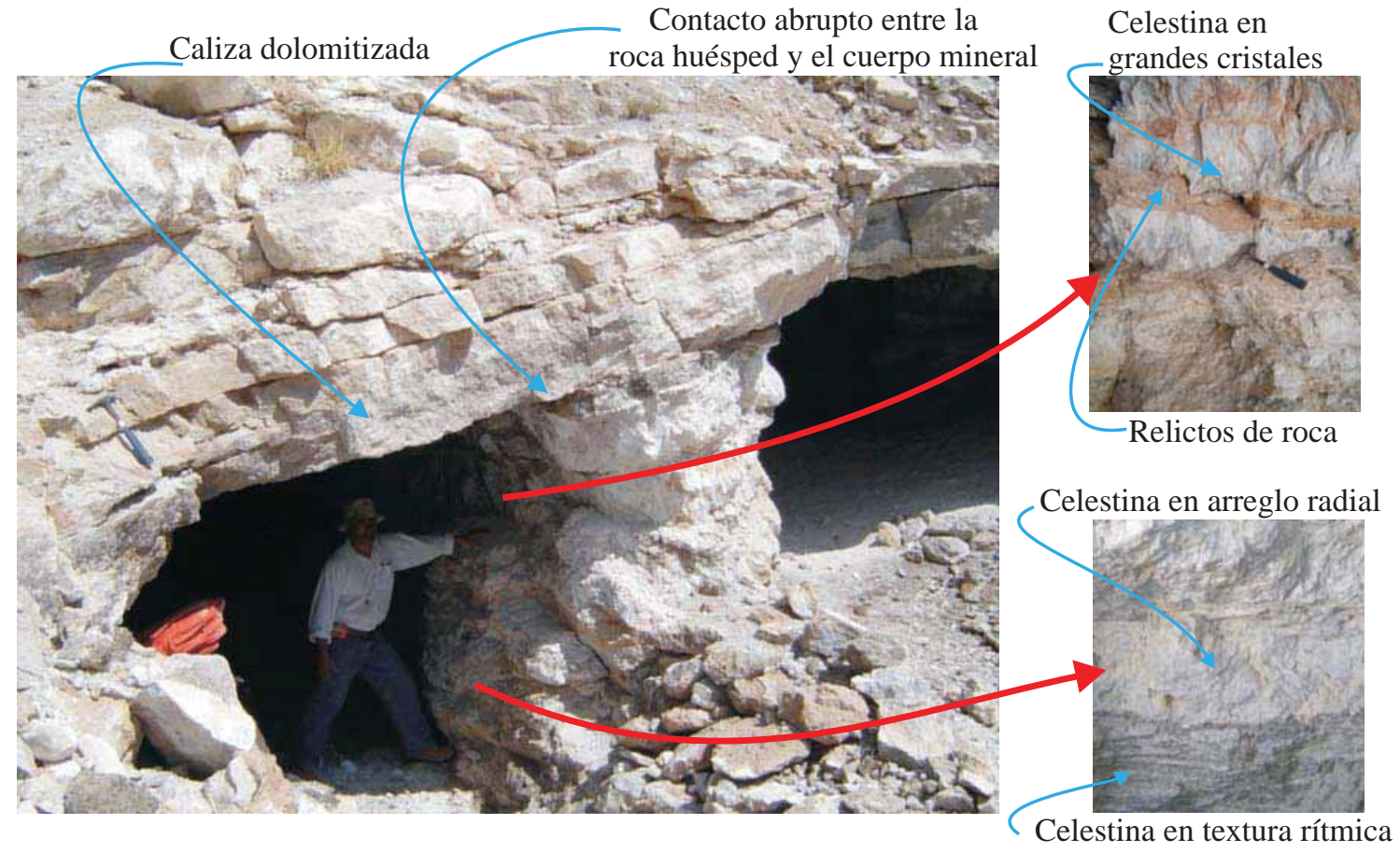

Figura 12. Fotografías mostrando los contactos abruptos entre las rocas encajonantes y las mineralizaciones estratoligadas, con relictos de la roca encajonante que se distribuyen dentro de los cuerpos mineralizados de forma paralela a la roca hospedante, y mostrando las texturas predominantes en los cuerpos mineralizados de la Sierra de Los Alamitos, Coahuila (fotografías cortesía de Aldo Ramos Rosique).

de cuenca, y eventualmente concentrado en los numerosos depósitos de celestina en la porción sur de esta región. En este sentido, la escasa presencia de mineralizaciones de celestina sobre la Península Burro - Peyotes (rica, en cambio, en depósitos de fluorita) estaría explicada por el predominio de fragmentos detríticos de origen metasedimentario en las formaciones clásticas y su poca o nula generación de estroncio radiogénico.

\section{Conclusiones}

En el estado de Coahuila y áreas circundantes se conocen más de 200 depósitos estratopligados de celestina, barita, fluorita y $\mathrm{Pb}-\mathrm{Zn}$ tipificados en este trabajo como yacimientos MVT (Mississippi Valley Type). Estos depósitos están hospedados en unidades estratigráficas características en la cuenca mesozoica de la región, cuya geometría fue definida por fosas y pilares tectónicos formados durante la segmentación del basamento durante el Permo-Triásico. Los pilares tectónicos (Bloque de Coahuila, Península Burro - Peyotes, Isla de La Mula e Isla de Monclova) constituyeron las tierras emergidas durante parte del Mesozoico y controlaron parcialmente la sedimentación de la cuenca a la cual servían de límite.

Los depósitos de celestina se hospedan en evaporitas y carbonatos de las Formaciones La Virgen (Barremiano) y Acatita (Albiano), mientras que los depósitos de fluorita se alojan en formaciones del Grupo Washita (Cenomaniano), equivalentes a la Formación Aurora, específicamente en el contacto de las lutitas de la Formación Del Río y las calizas arcillosas de la Formación Buda. Los depósitos de barita se hospedan en rocas evaporíticas de la Formación La Virgen (Barremiano) y carbonatos de plataforma de la formación Cupidito (Aptiano Inferior-Medio), mientras que los depósitos de $\mathrm{Pb}-\mathrm{Zn}$ se encajonan en rocas evaporíticas y carbonatadas de plataforma de las Formaciones Cupidito, Cupido, Aurora y La Virgen, con edades del Barremiano al Cenomaniano. De esta manera, los depósitos de celestina y fluorita se localizan en las secciones estratigráficas más jóvenes de la cuenca mesozoica, y los depósitos de $\mathrm{Pb}-\mathrm{Zn}$, si bien se encuentran en secciones estratigráficas similares, también se han encontrado en formaciones más antiguas.

La mayoría de los depósitos MVT del Noreste de México se localizan sobre los pilares tectónicos o cerca a sus límites, como lo ilustran los depósitos de celestina y $\mathrm{Pb}-\mathrm{Zn}$ sobre el Bloque de Coahuila, los depósitos de fluorita sobre la Península de Burro - Peyotes, y los depósitos de barita-Pb-Zn en las cercanías de la Isla de La Mula.

Estos depósitos en su conjunto definen una provincia metalogenética, propuesta formalmente por primera vez como Provincia MVT del Noreste de México (PMNM). Esta provincia está definida en base a la similitud de la configuración geológica y litologías a las cuales los depósitos minerales están asociados, así como a su localización en áreas y en secciones estratigráficas específicas 


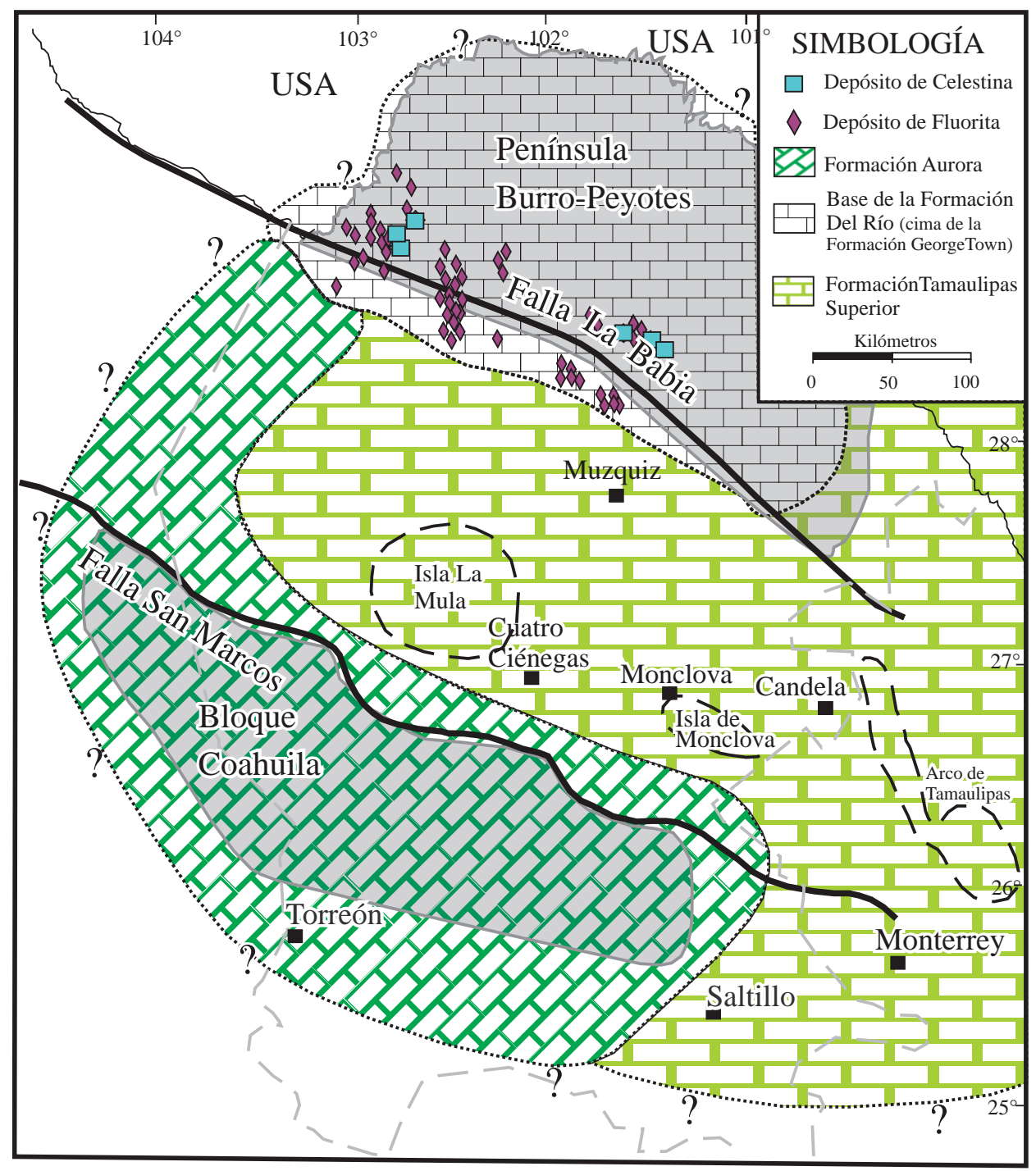

Figura 13. Distribución de las mineralizaciones de fluorita (rombos) y celestina (cruces) en la base de la Formación Buda (Cenomaniano Medio), con los rasgos paleogeográficos de la época. Modificado de Eguiluz de Antuñano (2001).

(especialmente con respecto a los altos paleogeográficos y fallas reactivadas). La distribución regional zonificada de los depósitos con mineralogía característica en la PMNM permite definir cuatro subprovincias: la Subprovincia Sur de Celestina, la Subprovincia Central de Pb-Zn, la Subprovincia Central de Barita y la Subprovincia Norte de Fluorita.

Se postula que el Sr y el Ba que formaron los depósitos de celestina y barita fueron lixiviados principalmente de las rocas clásticas y que la abundancia de los depósitos de celestina en la Subprovincia Sur de Celestina es debida a la abundancia de $\mathrm{Sr}$ radiogénico de las rocas clásticas formadas por detritos de basamento granítico permo-triásico del Bloque de Coahuila. En el lado norte de la cuenca mesozoica (Subprovincia Norte de Fluorita), las rocas metasedimentarias permo-triásicas fueron la fuente de las rocas clásticas mesozoicas, las cuales aportaron mucho menos $\mathrm{Sr}$ radiogénico que en el caso anterior, de tal manera que sólo se formaron depósitos de tipo MVT de fluorita.

\section{Agradecimientos}

El presente trabajo ha contado con el financiamiento del proyecto IN 102 107-3 de DGAPA-PAPIIT (UNAM), del proyecto CONACyT 58825 y con el presupuesto de asignaciones anuales a investigadores del Centro de Geociencias. Agradecemos el apoyo logístico durante el trabajo de campo del personal de la oficina en Saltillo (Coahuila) del Servicio Geológico Mexicano (SGM), especialmente a Carlos Martínez Ramos y Carlos Rivera Martínez y, muy especialmente, a todo el personal 


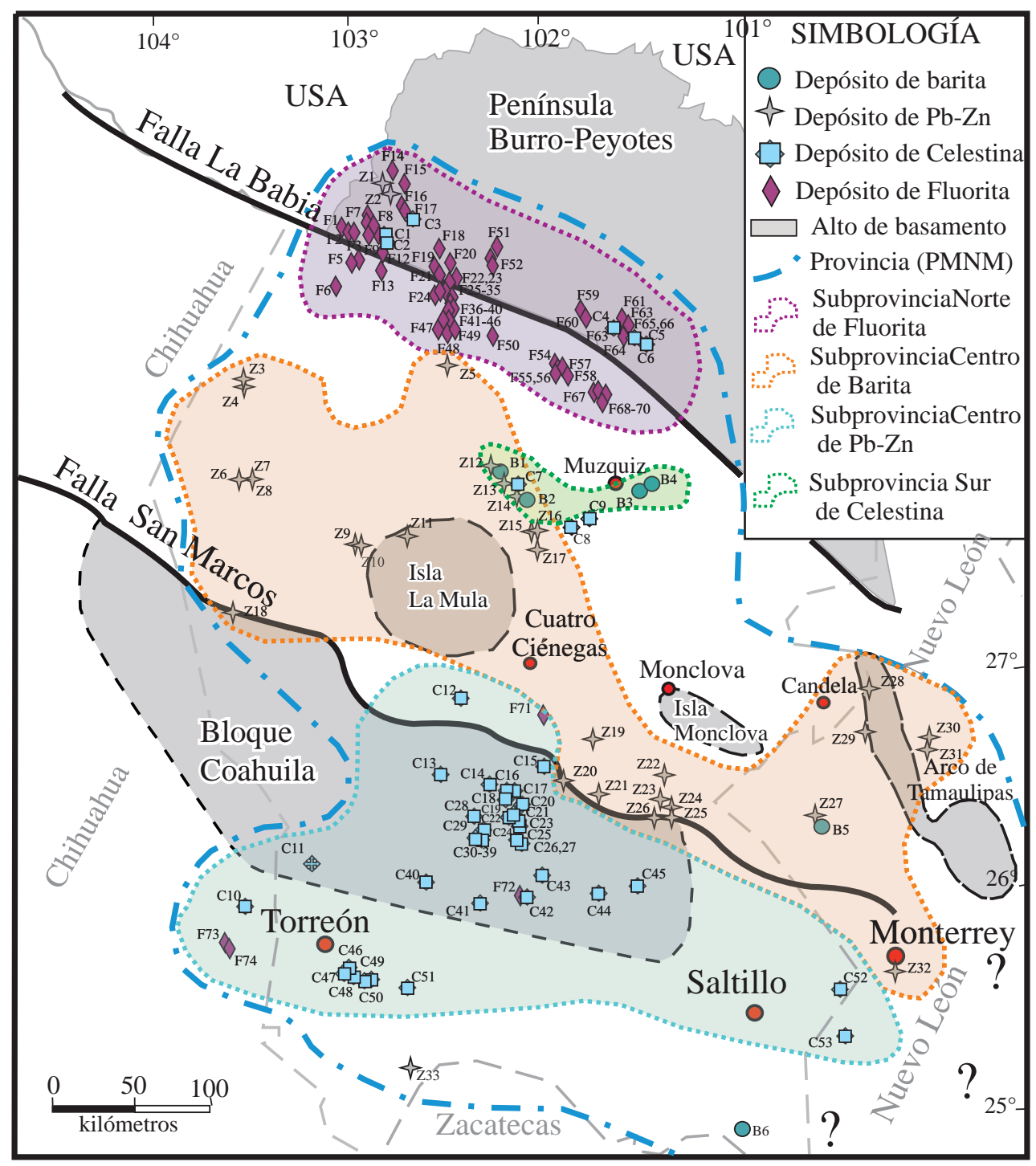

Figura 14. Delimitación de la Provincia Metalogenética MVT del Noreste de México (PMNM) y sus cuatro subprovincias. Elementos paleogeográficos tomados de Chávez-Cabello et al. (2005). Depósitos de barita (círculos verdes): (B1) Sierra de Santa Rosa (Barosa); (B2) San Juan; (B3) María Elena, El Palmito, Santa Rosa, El Potrero; (B4) El Cedral; (B5) Mina; (B6) La Luz. Depósitos de Zn-Pb (estrellas rojas): (Z1) Puerto Rico; (Z2) Carmen; (Z3) Diamantina; (Z4) San Lorenzo; (Z5) Cerritos I and II; (Z6) Viky, Santa Elena, La Cruz; (Z7) La Esperanza; (Z8) Dos Hermanos; (Z9) San Francisco; (Z10) Puerto Arturo; (Z11) La Bayoneta; (Z12) El Porvenir; (Z13) Carrizalejo; (Z14) Las Águilas; (Z15) Cedro I; (Z16) Las Torres, Las Torres I; (Z17) El Cedro; (Z18) Agrupamiento Fortaleza; (Z19) Agrupamiento Reforma; (Z20) La Luz; (Z21) Nueva Reforma; (Z22) Roca Verde; (Z23) Roca Flores; (Z24) Roca Rica, San Eugenio, Rama Azul; (Z25) Rincón Rojo; (Z26) Bonanza; (Z27) Agrupamiento Mina; (Z28) Sacramento; (Z29) Minas Viejas; (Z30) Nuevo México, La Carmencita; (Z31) La Cucaracha; (Z32) El Diente, Victoria I and II; (Z33) El Socorro. Depósitos de celestina (cruces azules): (C1) Oasis; (C2) Elvia; (C3) Max II; (C4) El Gari, El Cuadrangular; (C5) El Tule; (C6) La Victoría; (C7) Santa María; (C8) Las Peñitas; (C9) La Noria; (C10) Pirámide III; (C11) La Candelaria; (C12) Angélica; (C13) El Lucero; (C14) San Marcos; (C15) Blanquita; (C16) San José; (C17) Sotolito; (C18) Ocotillo; (C19) San Fernando; (C20) El Quemado; (C21) Ampliación San Marcos, Prospecto 5; (C22) Australia; (C23) Montejano I; (C24) Montejano II; (C25) El Latrisco; (C26) La Bola; (C27) La Carroza; (C28) La Tinaja; (C29) San Lorenzo; (C30) El Venado; (C31) Campo Patricio 2; (C32) Campo Patricio 1; (C33) El Diablo; (C34) El Volcán; (C35) Campo Patricio 4; (C36) Campo Patricio 3; (C37) Campo Patricio 5; (C38) La Chenta Norte; (C39) La Chenta Sur; (C40) El Caviar; (C41) La Guadalupana; (C42) La Ilusión; (C43) Del Rincón; (C44) La Yesuda; (C45) San Agustín; (C46) La Discordia; (C47) San Lorenzo 3; (C48) San Luis; (C49) Santo Tomás; (C50) Cerro Bola; (C51) La Milagrosa; (C52) La Flor; (C53) Ampliación La Flor. Depósitos de fluorita (rombos morados): (F1) San Genaro, Evelyn, Evelyn 2; (F2) Tres Hermanos; (F3) Ponchito; (F4) La Bonita; (F5) Josefinas; (F6) Navideño 4; (F7) Ima, Último; (F8) Totopos 1; (F9) Oasis 4; (F10) Aries 1; (F11) Peñón Blanco; (F12) El Otomí; (F13) Osiris, (F14) Santa Anita; (F15) Santa Anita 2; (F16) Un Día De Estos 1 and 3; (F17) El Jardín; (F18) Minas Fronterizas; (F19) Lorena; (F20) San Felipe; (F21) Nueva York; (F22) Santa María; (F23) La Victoria; (F24) Susana; (F25) Las Indias; (F26) Bonanza; (F27) Alba Iris; (F28) Pandita, Tohui; (F29) San Cristóbal; (F30) Los Fresnos; (F31) Cinco Hermanos; (F32) Arquímedes; (F33) Europa; (F34) Graciela; (F35) Las Carmelas; (F36) Navidad, Año Nuevo; (F37) San Cachito; (F38) Kentucky; (F39) María; (F40) San Pedro; (F41) San Miguel 1; (F42) Los Buras; (F43) El Número Nueve; (F44) Cerro Colorado; (F45) La Paloma; (F46) El Patrón; (F47) La Purísima - El Paso; (F48) Los Cuates; (F49) Fátima; (F50) La Güera, La Güera 1, La Gorriona 3; (F51) Amigos; (F52) Gaby; (F53) Ataco; (F54) El Alto; (F55) Rosalba; (F56) San Roberto 2; (F57) San José; (F58) San Rafael, San Rafael 1; (F59) El Güero, El Güero 1; (F60) Camarón 2, Camarón 14; (F61) Alcón 0-3; (F62) El Gari, El Cuadrangular, Alcón 0-4; (F63) Las Delias; (F64) Tayoltita; (F65) La Macarena, Valencia; (F66) La Muralla; (F67) Sofito Tercero; (F68) Chubasco 1, Chubasco 2; (F69) La Mariposa 1, 2,3 and 4; (F70) San Antonio; (F71) Sierra San Marcos, Pinos (La Becerra); (F72) La Reina, Los Amigos; (F73) Eva 13; (F74) Margarita 4. 
del Centro Documental de Recursos Minerales del SGM por su espléndida ayuda en la consulta y manejo del alrededor de 500 reportes mineros que fueron examinados durante nuestra investigación. Durante nuestro trabajo de campo inicial contamos con la inestimable guía de Antonio González Ramos y el apoyo adicional de Agustín Rodríguez Santos, Hugo Martínez, Arturo Cantú y Cantú, Fausto Cantú Arocha, Gilles Levresse, y Jordi Tritlla. La localización de algunos depósitos fue posible gracias a Amador Núñez. Este trabajo se ha beneficiado ostensiblemente de las revisiones críticas de Elena Centeno García, Martín Valencia Moreno y Lucas Ochoa Landín.

\section{Referencias}

Aguayo, C.J.E., 1978, Facies sedimentarias y diagénesis de la Formación Novillo (Jurásico Superior), noreste de México: Revista del Instituto Mexicano del Petróleo, X (4), 5-45.

Anderson, T.H., Schmidt, V.A., 1983, The evolution of Middle America and the Gulf of Mexico-Caribbean region during Mesozoic time: Geological Society of America Bulletin, 94, 941-966.

Charleston, S., 1981, A summary of the structural geology and tectonics of the state of Coahuila, Mexico, en Smith, C.I. (ed.), Lower Cretaceous stratigraphy and structure northern Mexico: West Texas Geological Society Publication, 81-74, 28-36.

Chávez-Cabello, G., Aranda-Gómez, J.J., Molina-Garza, R.S., CossíoTorres, T., Arvizu-Gutiérrez, I.R., González-Naranjo, G.A., 2005, La falla San Marcos: una estructura jurásica de basamento multirreactivada del noreste de México: Boletín de la Sociedad Geológica Mexicana, 57, 27-52.

Eguiluz de Antuñano, S., 2001, Geologic evolution and gas resources of the Sabinas Basin in Northeastern Mexico, en Bartolini, C., Buffler, R.T., Cantú-Chapa, A. (eds.), The western Gulf of Mexico Basin: Tectonics, sedimentary basins, and petroleum systems: American Association of Petroleum Geologists Memoir, 75, 241-270.

Fastovsky, D.E.O., Hermes, D., Strater, N.H., Bowring, S.A., Clark, J.M., Montellano, M., Hernández, R., 2005, Pre-Late Jurassic, fossilbearing volcanic and sedimentary red beds of Huizachal Canyon, Tamaulipas, Mexico, en Anderson, T.H., Nourse, J.A., McKee, J.W., Steiner, M.B. (eds.), The Mojave-Sonora megashear hypothesis: Development, assessment, and alternatives: Geological Society of America Special Paper, 393, 259-282

Garrison, J.M., McMillan, N.J., 1999, Evidence for Jurassic continentalrift magmatism in NE Mexico: Allogenic metaigneous blocks in El Papalote evaporite diapir, La Popa Basin, Nuevo Leon, Mexico, en Bartolini, C., Wilson, J.L., Lawton, T.F. (eds.), Mesozoic sedimentary and tectonic history of north-central Mexico: Boulder, Colorado, EUA, Geological Society of America Special Paper, 340, 319-332.

Goldhammer, R.K., 1999, Mesozoic sequence stratigraphy and paleogeographic evolution of northeast Mexico, en Bartolini, C., Wilson, J.L., Lawton, T.F. (eds.), Mesozoic sedimentary and Tectonic History of North-Central Mexico: Boulder, Colorado, EUA, Geological Society of America Special Paper, 340, 1-58.

González-Partida, E., Carrillo-Chávez, A., Grimmer, J.O.W., Pironon, J., Mutterer, J., Levresse, G., 2003, Fluorite deposits at EncantadaBuenavista, Mexico: products of Mississippi Valley type processes: Ore Geology Review, 23, 107-124.

Humphrey, W.E., 1956, Tectonic framework of northeast Mexico: Gulf Coast Association of Geologists, Society Transactions, 6, 25-35.

Imlay, R.W., 1936, Evolution of the Coahuila Peninsula, Mexico, Part IV, Geology of the western part of the Sierra de Parras: Geological Society of America Bulletin, 47, 1091-1152.

Imlay, R.W., 1940, Neocomian faunas of northern Mexico: Geological Society of America Bulletin, 51, 117-190.
Johnson, C.A., 1989, Structural Analysis of the fold and thrust belt in the vicinity of Monterrey, northeastern Mexico: Houston, Texas, EUA, Exxon Productions Research Company Report (inédito), $40 \mathrm{p}$.

Johnson, C.R., Ward, W.C., Goldhammer, R.K., 1991, Mechanisms for high-frequency cyclicity in the Upper Jurassic limestones of northeastern Mexico: American Association of Petroleum Geologists Bulletin, 75, 603.

Jones, N.W., McKee, J.W., Márquez, D.B., Tovar, J., Long, L.E., Laudon, T.S., 1984, The Mesozoic La Mula Island, Coahuila, Mexico: Geological Society of America Bulletin, 95, 1226-1241.

Kesler, S.E. Jones, L.M., 1981, Sulfur and strontium isotopic geochemistry of celestite, barite and gypsum from the Mesozoic basins of Northeastern Mexico: Chemical Geology, 31, 211-224.

Kisvaransayi, G., Grant, S.K., Pratt, W.P., Koening, J.W. (eds.), 1983, International Conference on Mississippi Valley-Type Lead-Zinc Deposits, Proceedings Volume: Rolla, Missouri, EUA, University of Missouri-Rolla, $603 \mathrm{p}$.

Leach, D.L., Sangster, D.F., 1993, Mississippi Valley-type lead-zinc ore deposits, en Kirkham, R.V., Sinclair, W.D., Thorpe, R.I., Duke, J.M. (eds.), Mineral Deposit Models, Geological Association of Canada, Special Paper, 40, 289-314.

Lehmann, C., Osleger, D.A., Montañez, I.P., Sliter, W., Arnaud-Vanneau, A., Banner, J., 1999, Evolution of Cupido and Coahuila carbonate platforms, Early Cretaceous, northeastern Mexico: Geological Society of America Bulletin, 111, 1010-1029.

López, R., Cameron, K.L., Jones, N.W., 2001, Evidence for Paleoproterozoic, Grenvillian, and Pan-African age Gondwanan crust beneath northeastern Mexico: Precambrian Research, 107, 195-214.

McKee, W.J., Jones, W.N., Anderson, H.T., 1988, Las Delicias Basin, a record of Late Paleozoic arc volcanism in northeastern Mexico: Geology, 16, 37-40.

Ohle, E.L., 1959, Some considerations on determining the origin of ore deposits of Mississippi Valley-type: Economic Geology, 54, 769-789.

Oivanki, S.M., 1974, Paleodepositional environments in the Upper Jurassic Zuloaga Formation (Smackover), northeast Mexico: Gulf Coast Association of Geologists, Society Transactions, 24, 258-278.

Padilla y Sánchez, R.J., 1986a, Geologic evolution of the Sierra Madre Oriental between Linares, Concepción del Oro, Saltillo, and Monterrey, Mexico: Austin, Texas, EUA, The University of Texas at Austin, Tesis de doctorado inédita, $217 \mathrm{p}$.

Padilla y Sánchez, R.J., 1986b, Post-Paleozoic Tectonics of Northeast Mexico and its role in the evolution of the Gulf of Mexico: Geofísica Internacional, 25, 157-206.

Pindell, J.L., 1985, Alleghenian reconstructions and subsequent evolution of the Gulf of Mexico, Bahamas, and proto-Caribbean: Tectonics, 4, 1-39.

Puente-Solís, R., 2006, Distribución de los depósitos estratoligados de barita, celestina, fluorita y plomo-zinc en el noroeste de México y ejemplo de los mantos de celestita de la Sierra del Venado, Coahuila: Juriquilla, Querétaro, México, Programa de Posgrado en Ciencias de la Tierra, Universidad Nacional Autónoma de México, Tesis de maestría inédita, $180 \mathrm{p}$.

Ramírez, C.J., 1966, Tabla de correlación estratigráfica Zona noreste: Archivos de Pemex, reporte inédito.

Rueda-Gaxiola, J., López-Ocampo, E., Dueñas, M.A., Rodríguez, J.L., Torres-Rivero, A., 1999, The palynological method: basis for defining stratigraphy and age of the Los San Pedros Allogropu, Huizachal-Peregrina anticlinorium, Mexico, en Bartolini, C., Wilson, J., Lawton, T. (eds.), Mesozoic sedimentary and tectonic history of North-Central Mexico: Boulder, Colorado, EUA, Geological Society of America Special Paper, 340, 229-269.

Salvador, A., Green, A., 1980, Opening of the Caribbean Tethys (Origin and development of the Caribbean and the Gulf of Mexico), en Auboin, J., et al. (eds.), Géologie des chaînes alpines issues de la Téthys, Bureau de Recherche Géologique et Minière, Mémoires, $115,224-229$.

Sangster, D.F., 1983, Mississippi Valley-Type deposits: a geological 
mélange, en Kisvaransayi, G., Grant, S. K., Pratt, W.P., Koening, J.W. (eds.), International Conference on Mississippi Valley-Type Lead-Zinc Deposits, Proceedings Volume: Rolla, Missouri, EUA, University of Missouri-Rolla, 7-19.

Sverjensky, D.A., 1986, Genesis of Mississippi Valley-type lead-zinc deposits: Annual Reviews of Earth and Planetary Sciences, 14, 177-199.

Tinker, S.W., 1985, Lithostratigraphy and biostratigraphy of the Apitan La Peña Formation, northeast México and south Texas (part I), and the depositional setting of the Aptian Pearsall-La Peña Formations, Texas subsurface and northeast Mexico: Why is there not another Fairway Field? (part 2): Ann Arbor, University of Michigan, tesis de maestría inédita, $80 \mathrm{p}$.

Vinet, M.J., 1975, Geology of the Sierra of Balauartes and Sierra de Pajaros Azules: New Orleans, Louisiana, EUA, Louisiana State University, Tesis de doctorado inédita, $124 \mathrm{p}$.

Wilson, J.L., 1990, Basement structural controls on Mesozoic carbonates facies in northeastern Mexico: A review, en Tucker, M.E., Wilson, J.L., Crevello, P.D., Sarg, J.R., Read, J.F. (eds.), Carbonate platforms, facies, sequences and evolution: International Association of Sedimentologists, Special Publication, 9, 235-255.

Wilson, J.L., Pialli, G., 1977, A Lower Cretaceous shelf margin in Northern Mexico, en Cretaceous carbonates of Texas and Mexico: Applications to subsurface exploration: Austin, Texas, EUA, The University of Texas at Austin, Bureau of Economic Geology, Report of Investigations, 89, 286-298
Wilson, J.L., Ward, W.C., Finneran, J. (eds.), 1984, A field guide to Upper Jurassic and Lower Cretaceous carbonate platform and basin systems, Monterrey-Saltillo area, northeast Mexico: Gulf Coast Section, Society of Economic Paleontologists and Mineralogists, $76 \mathrm{p}$.

Winker, C.D., Buffler, R.T., 1988, Paleogeographic evolution of early deep-water Gulf of Mexico and margins, Jurassic to Middle Cretaceous (Comanchean): American Association of Petroleum Geologists Bulletin, 72, 318-346.

Manuscrito recibido: Diciembre 12, 2006

Manuscrito corregido recibido: Enero 18, 2007

Manuscrito aceptado: Febrero 7, 2007 